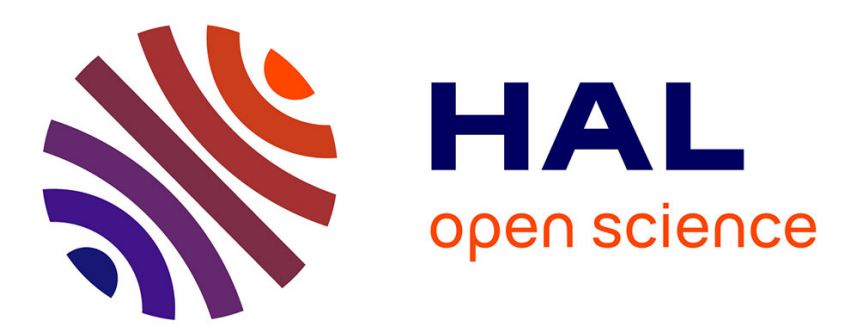

\title{
A domain decomposition method to couple nonisothermal compositional gas liquid Darcy and free gas flows
}

\author{
N Birgle, R. Masson, L Trenty
}

\section{- To cite this version:}

N Birgle, R. Masson, L Trenty. A domain decomposition method to couple nonisothermal compositional gas liquid Darcy and free gas flows. Journal of Computational Physics, 2018, 10.1016/j.jcp.2018.04.035 . hal-01584887v2

\section{HAL Id: hal-01584887 \\ https://hal.science/hal-01584887v2}

Submitted on 19 Apr 2018

HAL is a multi-disciplinary open access archive for the deposit and dissemination of scientific research documents, whether they are published or not. The documents may come from teaching and research institutions in France or abroad, or from public or private research centers.
L'archive ouverte pluridisciplinaire HAL, est destinée au dépôt et à la diffusion de documents scientifiques de niveau recherche, publiés ou non, émanant des établissements d'enseignement et de recherche français ou étrangers, des laboratoires publics ou privés. 


\title{
A domain decomposition method to couple nonisothermal compositional gas liquid Darcy and free gas flows
}

\author{
N. Birgle*, R. Masson $\dagger^{\prime}$ L. Trenty
}

April 19, 2018

\begin{abstract}
A domain decomposition algorithm is introduced to couple nonisothermal compositional gas liquid Darcy and free gas flow and transport. At each time step, our algorithm solves iteratively the nonlinear system coupling the nonisothermal compositional Darcy flow in the porous medium, the RANS gas flow in the free-flow domain, and the transport of the species and of energy in the free-flow domain. In order to speed up the convergence of the algorithm, the transmission conditions at the interface are replaced by Robin type boundary conditions. The Robin coefficients are obtained from a diagonal approximation of the Dirichlet to Neumann operator related to a simplified model in the neighbouring subdomain. The efficiency of our domain decomposition algorithm is assessed on several test cases focusing on the modeling of the mass and energy exchanges at the interface between the geological formation and the ventilation galleries of geological radioactive waste disposal.
\end{abstract}

\section{Introduction}

Modeling the exchange processes at the interface between a free flow and a flow in a porous medium appears in a wide range of applications from food processing [1], wood or paper production [2], salinization of agricultural land [3], prediction of convective heat and moisture transfer at exterior building surfaces [4], to the study of the mass and energy exchanges at the interface between a nuclear waste disposal and the ventilation galleries $[5,6]$. This latter is the main focus of this paper in terms of application.

To model such physical processes, one needs to account, in the porous medium, for the flow of the liquid and gas phases including the vaporization of the water component in the gas phase and the dissolution of the gaseous components in the liquid phase. In the free-flow region, only the gas phase is considered assuming that the liquid phase is instantaneously vaporized at the interface. The transport of vapor in the gas free flow plays a crucial role to account for the change of the relative humidity which has a strong feedback on the evaporation rate at the interface. In such drying processes, the energy conservation must be taken into account in both domains since the temperature variations have a strong influence on the vaporization. These temperature variations are induced by the vaporization of the liquid phase itself or by differences of temperatures between the porous medium and the gas in the free-flow region. In our targeted application to deep geological disposal for

\footnotetext{
${ }^{*}$ Université Côte d'Azur, Inria, CNRS, Laboratoire J.A. Dieudonné, Team Coffee, Parc Valrose, 06108 Nice cedex 02, France, nabil.birgle@unice.fr

${ }^{\dagger}$ Université Côte d'Azur, Inria, CNRS, Laboratoire J.A. Dieudonné, Team Coffee, Parc Valrose, 06108 Nice cedex 02, France, roland.masson@unice.fr

${ }_{\ddagger}^{\ddagger}$ Andra, 1-7 rue Jean Monnet 92290 Chatenay-Malabry, France, laurent.trenty@andra.fr
} 
radioactive wastes, it is essential to consider the coupling effect between thermal energy produced by high level radioactive wastes and the porous medium desaturation.

A coupled model has been proposed in $[7,8]$ using matching conditions at the interface between the porous-medium and the free-flow regions. These coupling conditions state the continuity of the component molar and energy normal fluxes taking into account the instantaneous vaporization of the liquid phase, the continuity of the gas molar fractions and of the temperature, the continuity of the normal component of the normal stress, and the liquid gas thermodynamical equilibrium. In our case, the Beavers-Joseph condition [9] used in [7, 8] will be replaced by a no slip condition due to the low permeability of the porous medium.

In order to solve such coupled models, sequential algorithms based on Dirichlet-Neumann transmission conditions at the interface are frequently used (see [10,11] and the review [4]). As mentioned in $[10,11]$, the stability of these sequential algorithms requires very small time steps at the scale of the free flow leading to very large CPU times. To obtain an efficient algorithm, one needs to be able to use time steps at the scale of the porous medium with a quasi-stationary computation of the free flow at each time step. A time splitting algorithm with local time stepping in the free-flow domain is investigated in [12] for a related but different problem coupling the Richards equation in the porous medium and the Stokes equation for the liquid phase in the free-flow region.

Alternatively, fully coupled algorithms such as the ones developed in $[8,13,14,15]$ have been introduced, but they lead to nonlinear and linear systems which are difficult and expensive to solve since they do not take advantage of the different levels of coupling in the nonlinear system and prevent the use of on-the-shelves preconditioners.

The algorithm proposed in [5] for isothermal models is based on a splitting between (i) the Darcy model coupled with the transport in the free-flow region, and (ii) the flow in the free-flow region. Its efficiency comes from the weak coupling between the Darcy model and the free-flow while the coupling between the Darcy model and the transport in the free-flow region is strong.

Heterogeneous domain decomposition methods have been applied to couple different physical models in different parts of the domain. A coupled problem related to our model and which has been extensively addressed in the literature is the coupling of a single phase Darcy flow with a Stokes or Navier-Stokes free flow. For this type of model, different domain decomposition methods have been developed such as Robin Robin domain decomposition methods in [16], [17] or iterative substructuring domain decomposition algorithms (see the review [18]).

It is worth to mention that our coupled model somehow includes the Darcy - Navier-Stokes coupling for the common gas phase between the two regions. However this is not the dominant coupling in our model since the porous medium is assumed to weakly perturbate the gas velocity and pressure in the free-flow domain. Our strategy is based on the assumption that the dominant coupling is rather between (a) in the porous medium and (b) in the free-flow domain with

(a) the liquid pressure and the temperature in the porous medium governed approximately by the Richards equation and the energy conservation equation,

(b) the vapor molar fraction and the temperature in the free-flow domain governed approximately by the transport equations at fixed velocity and gas pressure.

These assumptions lead to the domain decomposition algorithm proposed in this work and based on optimized Schwarz methods. This algorithm solves iteratively at each time step until convergence to the fully coupled solution:

(i) the nonisothermal liquid gas Darcy flow in the porous-medium domain using at the interface Robin boundary conditions as well as an additional Dirichlet boundary condition for the gas pressure, 
(ii) the free-flow model for the velocity and for the pressure in the free-flow domain with a Dirichlet boundary condition for the velocity

(iii) the molar and energy transport in the free-flow domain with Robin boundary conditions.

The computation of the Robin coefficients of steps (i) and (iii) is crucial to guarantee the efficiency and robustness of the algorithm. This computation is based on a low frequency diagonal approximation of the Dirichlet to Neumann operator related to the neighboring subdomain model. In both cases, the methodology is first to simplify and linearize the neighboring subdomain problem and then to compute a diagonal approximation of its Dirichlet to Neumann operator which is exact on constant modes along the interface. Thanks to the quasi linearity of the free-flow transport model and to the small free-flow velocity and pressure perturbation, a rather accurate approximation can be computed for the Robin coefficients of step (i) while for step (iii) a rather rough zeroth-order Taylor approximation of the Dirichlet to Neumann operator is used which suffices to obtain a good order of magnitude. The combination of both approximations is shown in the numerical section to provide an efficient and robust convergence of the domain decomposition algorithm.

The outline of the remaining of this paper is the following. The coupled model and its formulation is detailed in section 2. The domain decomposition method used to solve the coupled nonlinear system at each time step, after Euler implicit time integration, is presented in section 3. The computation of the Robin coefficients to speed up the convergence of the domain decomposition algorithm is explained in section 4. The robustness and efficiency of our domain decomposition algorithm is assessed on four two-dimensional test cases presented in section 5 and including a wide range of model or discretization parameters such as the mesh size, the input free-flow velocity, the temperature range, the permeability, the capillary pressure and the heterogeneities. The first three test cases are defined by Andra and are related to the simulation of the mass and energy exchanges occurring at the interface between the geological formation and the ventilated excavated galleries.

\section{Formulation of the coupled model}

Let us denote by $\Omega_{\mathrm{pm}}$ the porous-medium domain, by $\Omega_{\mathrm{ff}}$ the free-flow domain and by $\Gamma=\partial \Omega_{\mathrm{pm}} \cap \partial \Omega_{\mathrm{ff}}$ the interface.

Let $\mathcal{P}=\{g, \ell\}$ denote the set of gas and liquid phases assumed to be both defined by a mixture of components $i \in \mathcal{C}$ among which the water component denoted by $w$ which can vaporize in the gas phase, and a set of gaseous components $j \in \mathcal{C} \backslash\{w\}$ which can dissolve in the liquid phase.

Both the gas and the liquid phases can be present in the porous-medium domain while only the gas phase is assumed to be present in the free-flow domain. Each phase $\alpha \in \mathcal{P}$ is defined by its pressure $p^{\alpha}$, temperature $T$ and molar fractions $\mathbf{c}^{\alpha}=\left(c_{i}^{\alpha}\right)_{i \in \mathcal{C}}$. For each phase, $\alpha \in \mathcal{P}, \zeta^{\alpha}\left(p^{\alpha}, T, \mathbf{c}^{\alpha}\right)$ denotes its molar density, $\rho^{\alpha}\left(p^{\alpha}, T, \mathbf{c}^{\alpha}\right)$ its mass density, $\mu^{\alpha}\left(p^{\alpha}, T, \mathbf{c}^{\alpha}\right)$ its dynamic viscosity, $h^{\alpha}\left(p^{\alpha}, T, \mathbf{c}^{\alpha}\right)$ its molar enthalpy, and $e^{\alpha}\left(p^{\alpha}, T, \mathbf{c}^{\alpha}\right)=h^{\alpha}\left(p^{\alpha}, T, \mathbf{c}^{\alpha}\right)-\frac{p^{\alpha}}{\zeta^{\alpha}}$ its molar internal energy. For the gas phase, assuming an ideal mixture, the molar enthalpy is defined by

$$
h^{g}\left(p^{g}, T, \mathbf{c}^{g}\right)=\sum_{i \in \mathcal{C}} c_{i}^{g} h_{i}^{g}\left(p^{g}, T\right)
$$

where $h_{i}^{g}\left(p^{g}, T\right)$ is the molar enthalpy of the component $i$ in the gas phase. For $i \in \mathcal{C}$ and $\alpha \in \mathcal{P}$, we also denote by $f_{i}^{\alpha}\left(p^{\alpha}, T, \mathbf{c}^{\alpha}\right)$ the fugacity of the component $i$ in the phase $\alpha$.

We now turn to the definition of the model in each subdomain followed by the description of the coupling conditions at the interface $\Gamma$. 


\subsection{Nonisothermal compositional Darcy flow in the porous medium}

Although the domain decomposition algorithm of section 3 can be written regardless of the choice of the primary unknowns in the porous-medium domain, it is convenient to specify this choice to fix ideas. Following $[19,20]$, our choice of the liquid gas Darcy flow formulation uses the pressures $p^{g}, p^{\ell}$ of both phases, the vector $\mathbf{f}=\left(f_{i}\right)_{i \in \mathcal{C}}$ of the component fugacities and the temperature, denoted by $T_{\mathrm{pm}}$ in the porous medium, as primary unknowns.

In this formulation, following [21], the molar fractions $\mathbf{c}^{\alpha}$ of each phase $\alpha \in \mathcal{P}$ are defined as a function of $p^{\alpha}, \mathbf{f}, T_{\mathrm{pm}}$ by inversion of the equations

$$
f_{i}^{\alpha}\left(p^{\alpha}, T_{\mathrm{pm}}, \mathbf{c}^{\alpha}\right)=f_{i}, \quad i \in \mathcal{C},
$$

which means that the molar fractions of an absent phase is extended by the molar fractions at equilibrium with the present phase. In addition, for each phase $\alpha \in \mathcal{P}$, the pressure $p^{\alpha}$ is also extended in the absence of the phase $\alpha$ in such a way that the closure law $\sum_{i \in \mathcal{C}} c_{i}^{\alpha}=1$ is always imposed (see $[19,20])$. Using this extension of the phase pressures, the gas saturation $s^{g}\left(\mathbf{x}, p^{g}-p^{\ell}\right)$ is defined by the inverse of the monotone graph extension of the capillary pressure function and the liquid saturation is given by $s^{\ell}=1-s^{g}$. Note that in the remaining of the paper, the molar fractions $\mathbf{c}^{\alpha}$ of each phase $\alpha \in \mathcal{P}$ are implicitely considered as the function $\mathbf{c}^{\alpha}\left(p^{\alpha}, T_{\mathrm{pm}}, \mathbf{f}\right)$ of the primary unknowns even if this dependence is not made explicit. This formulation is shown in [20] to be equivalent at the continuous level to more usual formulations not based on extensions of the phase molar fractions and based on alternative extensions of the phase pressures. At the discrete level, the extension of the phase molar fractions does not modify the discrete solution since the phase molar fractions are multiplied either by the phase saturation or by the phase mobility which both vanish when the phase if absent. On the other hand, the way the pressure of an absent phase is extended can clearly modify the flux at the interface between a single phase cell and a two phase cell. Nevertheless, convergence to the same solution is observed when the mesh is refined (see [20] for a comparison of different formulations and a more detailed discussion).

Let us define, for each component $i \in \mathcal{C}$, the total number of moles per unit pore volume by

$$
\mathrm{n}_{i}=\sum_{\alpha \in \mathcal{P}} \zeta^{\alpha} c_{i}^{\alpha} s^{\alpha}
$$

and the energy per unit volume by

$$
E=\phi(\mathbf{x}) \sum_{\alpha \in \mathcal{P}} \zeta^{\alpha} e^{\alpha} s^{\alpha}+(1-\phi(\mathbf{x})) \zeta^{r} e^{r}
$$

where $\phi(\mathbf{x})$ is the porous-medium porosity, $e^{r}\left(T_{\mathrm{pm}}\right)$ is the rock molar internal energy, and $\zeta^{r}\left(T_{\mathrm{pm}}\right)$ is the rock molar density.

The Darcy velocities are defined by

$$
\mathbf{u}^{\alpha}=-\frac{k_{\mathrm{r}}^{\alpha}\left(\mathbf{x}, s^{\alpha}\right)}{\mu^{\alpha}} \mathbb{K}(\mathbf{x})\left(\nabla p^{\alpha}-\rho^{\alpha} \mathbf{g}\right), \quad \alpha \in \mathcal{P},
$$

where $\mathbb{K}(\mathbf{x})$ is the porous medium absolute permeability tensor, $k_{\mathrm{r}}^{\alpha}\left(\mathbf{x}, s^{\alpha}\right)$ is the phase $\alpha$ relative permeability, and $\mathbf{g}$ is the gravitational acceleration vector.

The component molar flow rate is defined by

$$
\mathbf{v}_{i}=\sum_{\alpha \in \mathcal{P}} \zeta^{\alpha} c_{i}^{\alpha} \mathbf{u}^{\alpha}-d_{\mathrm{pm}}^{\alpha}\left(\mathbf{x}, s^{\alpha}\right) \nabla c_{i}^{\alpha}, \quad i \in \mathcal{C},
$$

where $d_{\mathrm{pm}}^{\alpha}\left(\mathbf{x}, s^{\alpha}\right)$ is the effective diffusion coefficient of the phase $\alpha \in \mathcal{P}$ in the porous medium. It typically depends on the molecular diffusion coefficient, the porous medium porosity, a geometric 
factor known as the tortuosity and the phase saturation (see $[22,23])$. The energy flow rate is defined by

$$
\mathbf{v}_{\mathrm{e}}=\sum_{\alpha \in \mathcal{P}} \zeta^{\alpha} h^{\alpha} \mathbf{u}^{\alpha}-\lambda_{\mathrm{pm}}\left(\mathbf{x}, s^{\ell}\right) \nabla T_{\mathrm{pm}}
$$

where $\lambda_{\mathrm{pm}}\left(\mathbf{x}, s^{\ell}\right)$ is the thermal conductivity of the rock fluid mixture.

For a final time $t_{\mathrm{f}}$, the model using the primary unknowns $p^{g}, p^{\ell}, \mathbf{f}$ and $T_{\mathrm{pm}}$ accounts for the following mole and energy conservation equations

$$
\begin{aligned}
\phi \partial_{t} \mathrm{n}_{i}+\nabla \cdot \mathbf{v}_{i} & =0, & & \text { on } \Omega_{\mathrm{pm}} \times\left(0, t_{\mathrm{f}}\right), \quad i \in \mathcal{C}, \\
\partial_{t} E+\nabla \cdot \mathbf{v}_{\mathrm{e}} & =\mathrm{g}_{\mathrm{e}}(\mathbf{x}, t), & & \text { on } \Omega_{\mathrm{pm}} \times\left(0, t_{\mathrm{f}}\right),
\end{aligned}
$$

coupled with the sum to 1 of the molar fractions for each phase given by

$$
\sum_{i \in \mathcal{C}} c_{i}^{\alpha}=1, \quad \text { on } \Omega_{\mathrm{pm}} \times\left(0, t_{\mathrm{f}}\right), \quad \alpha \in \mathcal{P},
$$

where $\mathrm{g}_{\mathrm{e}}(\mathbf{x}, t)$ is an additional heat source term defined on $\Omega_{\mathrm{pm}} \times\left(0, t_{\mathrm{f}}\right)$.

\subsection{Flow and transport model in the free-flow domain $\Omega_{\mathrm{ff}}$}

To fix ideas, the primary unknowns in the free-flow domain are defined by the gas pressure denoted by $p$, the gas velocity denoted by $\mathbf{u}$, the gas molar fractions denoted by $\mathbf{c}=\left(c_{i}\right)_{i \in \mathcal{C}}$ and the temperature denoted by $T_{\mathrm{ff}}$. The gas flow and transport is described by a Reynolds Averaged Navier-Stokes (RANS) compositional and nonisothermal model. It is assumed to be quasi-stationary at the time scale of the porous medium and is governed by the following momentum conservation equation coupled with the mass and energy conservation equations

$$
\begin{aligned}
\nabla \cdot \mathbb{T} & =\rho^{g} \mathbf{g}, & & \text { on } \Omega_{\mathrm{ff}} \times\left(0, t_{\mathrm{f}}\right), \\
\nabla \cdot \mathbf{w}_{i} & =0, & & \text { on } \Omega_{\mathrm{ff}} \times\left(0, t_{\mathrm{f}}\right), \\
\nabla \cdot \mathbf{w}_{\mathrm{e}} & =0, & & \text { on } \Omega_{\mathrm{ff}} \times\left(0, t_{\mathrm{f}}\right),
\end{aligned}
$$

together with the closure law

$$
\sum_{i \in \mathcal{C}} c_{i}=1
$$

In (4), the following constitutive equations are used. The stress tensor is defined by

$$
\mathbb{T}=\rho^{g} \mathbf{u} \otimes \mathbf{u}-\left(\mu_{\mathrm{t}}+\mu^{g}\right)\left(\nabla \mathbf{u}+\nabla^{\mathrm{t}} \mathbf{u}\right)+p \mathbb{I},
$$

the component molar flow rate is defined by

$$
\mathbf{w}_{i}=\zeta^{g}\left(c_{i} \mathbf{u}-\left(d_{\mathrm{t}}+d^{g}\right) \nabla c_{i}\right),
$$

and the energy flow rate by

$$
\mathbf{w}_{\mathrm{e}}==\zeta^{g} h^{g} \mathbf{u}-\sum_{i \in \mathcal{C}} \zeta^{g} h_{i}^{g}\left(d_{\mathrm{t}}+d^{g}\right) \nabla c_{i}-\left(\lambda_{\mathrm{t}}+\lambda^{g}\right) \nabla T_{\mathrm{ff}}=\sum_{i \in \mathcal{C}} h_{i}^{g} \mathbf{w}_{i}-\left(\lambda_{\mathrm{t}}+\lambda^{g}\right) \nabla T_{\mathrm{ff}} .
$$

The standard thermal conductivity of the gas phase is denoted by $\lambda^{g}$ and the gas Fickian diffusion coefficient by $d^{g}$. They both can depend on $p, T_{\mathrm{ff}}$ and $c$ but will be taken constant in the numerical experiments. The turbulent dynamic viscosity $\mu_{\mathrm{t}}$ is typically obtained using an algebraic turbulent model or a more advanced $k-\epsilon$ model [15] from which is also deduced the turbulent diffusivity $d_{\mathrm{t}}$ and the turbulent thermal conductivity $\lambda_{\mathrm{t}}$. Note that, in the following numerical experiments, the turbulent dynamic viscosity, diffusivity and thermal conductivity will be computed from the stationary uncoupled gas flow. This is motivated by the small perturbation of the free-flow velocity and pressure induced by the coupling with the porous medium. In practice we will use the Prandtl algebraic turbulent model as in [5] (see the numerical Section 5). 


\subsection{Transmission conditions at the interface}

At the interface $\Gamma$ between the free-flow and the porous-medium domains, the coupling conditions are those stated in $[7,15,5]$ where we have replaced the Beaver Joseph condition by the simpler no slip condition due to the low permeability of the porous medium in our application.

Let $\mathbf{n}_{\mathrm{pm}}$ and $\mathbf{n}_{\mathrm{ff}}$ such that $\mathbf{n}_{\mathrm{pm}}+\mathbf{n}_{\mathrm{ff}}=0$ be the unit normal vectors at the interface $\Gamma$ oriented respectively outward from the porous-medium and free-flow domains. The interface conditions state the continuity of the gas molar fractions and of the molar normal flow rates, the continuity of the temperature and of the energy normal flow rate as well as the gas no slip condition in the free-flow domain and the continuity between the gas pressure in the porous-medium domain and the normal component of the normal stress in the free-flow domain.

$$
\begin{aligned}
& c_{i}^{g}=c_{i}, \quad \mathbf{v}_{i} \cdot \mathbf{n}_{\mathrm{pm}}=-\mathbf{w}_{i} \cdot \mathbf{n}_{\mathrm{ff}}, \quad \text { on } \Gamma \times\left(0, t_{\mathrm{f}}\right), \quad i \in \mathcal{C}, \\
& T_{\mathrm{pm}}=T_{\mathrm{ff}}, \quad \mathbf{v}_{\mathrm{e}} \cdot \mathbf{n}_{\mathrm{pm}}=-\mathbf{w}_{\mathrm{e}} \cdot \mathbf{n}_{\mathrm{ff}}, \quad \text { on } \Gamma \times\left(0, t_{\mathrm{f}}\right), \\
& \mathbf{u} \wedge \mathbf{n}_{\mathrm{ff}}=0, \quad p^{g}=\mathbf{n}_{\mathrm{ff}} \cdot \mathbb{T} \mathbf{n}_{\mathrm{ff}}, \quad \text { on } \Gamma \times\left(0, t_{\mathrm{f}}\right) .
\end{aligned}
$$

It is complemented by the sum to one of the liquid molar fractions in the porous-medium domain and of the gas molar fractions both in the porous-medium and free-flow domains.

$$
\sum_{i \in \mathcal{C}} c_{i}^{\alpha}=1, \quad \text { on } \Gamma \times\left(0, t_{\mathrm{f}}\right), \quad \alpha \in \mathcal{P} .
$$

It results from the first equation in (9) and from (10) that the equation $\sum_{i \in \mathcal{C}} c_{i}=1$ also holds on $\Gamma \times\left(0, t_{\mathrm{f}}\right)$. Note that, due to our choice ot the Darcy model formulation using the primary unknowns $p^{g}, p^{\ell}, \mathbf{f}, T_{\mathrm{pm}}$, the liquid gas equilibrium is a consequence of the equations (10) and of the gas saturation $s^{g}\left(\mathbf{x}, p^{g}-p^{\ell}\right)$ expressed as a function of $p^{g}-p^{\ell}$ using the inverse of the monotone graph extension of the capillary pressure. Note also that, in the absence of the gas phase at the interface on the porous-medium side, the extended gas pressure and gas molar fractions are used to express the continuity of the gas pressure and gas molar fractions.

\section{Domain decomposition algorithm}

The transmission conditions (9)-(10) at the interface $\Gamma$ couple the porous medium equations (2)-(3) with the free-flow domain equations (4)-(5). The system (2)-(10) is integrated in time using an Euler implicit scheme, which leads to solve at each time step a fully coupled nonlinear system. The solution of this nonlinear system is obtained at each time step using a domain decomposition algorithm solving iteratively until convergence the three following submodels:

(i) the nonisothermal compositional liquid gas Darcy flow in the porous-medium domain using at the interface Robin boundary conditions as well as a Dirichlet boundary condition for the gas pressure,

(ii) the RANS flow equations for the velocity and for the pressure in the free-flow domain with a Dirichlet boundary condition for the velocity at the interface,

(iii) the convection diffusion equations in the free-flow domain for the gas molar fractions and the temperature with Robin boundary conditions at the interface.

This approach has two advantages. Firstly it allows to use different codes for the porous-medium and the free-flow problems. Secondly, it reduces the complexity of the nonlinear and linear systems and make it possible to use on-the shelves preconditioners which results in a better efficiency compared with a monolithic Newton algorithm solving the fully coupled system [7, 15]. 
In the following, the time step count $n$ is omitted for the sake of clarity, and the component total number of moles and the total energy in the porous medium at the previous time step are denoted respectively by $\mathrm{n}_{i}^{n-1}$ and $E^{n-1}$. The domain decomposition count is denoted by the superscript $k$. As usual, the algorithm is initialized by the previous time step solution and by the initial condition at the first time step.

\subsection{Compositional Darcy flow with Robin boundary conditions}

The system (2)-(3) in the porous-medium domain is solved using Robin type boundary conditions at the interface $\Gamma$. These boundary conditions are obtained by linear combinations of, on the one hand, the continuity of the gas molar fractions and temperature, and, on the other hand, the continuity of the normal molar and energy flow rates. The coefficients of these linear combinations, denoted by $L$, $M_{i}, i \in \mathcal{C}$ and $N$ in (11), will be computed in subsection 4.1 as functions of $\mathbf{x}$ at the interface $\Gamma$ in order to provide a good low frequency diagonal approximation of the Dirichlet to Neumann operator related to the transport equations in the free-flow domain. The Robin coefficients $M_{i}, i \in \mathcal{C}$ introduce a coupling between the temperature and the molar fractions in the Robin boundary conditions which is necessary to obtain the convergence of the domain decomposition algorithm as exhibited in Figures 29 and 30 in the numerical section. This coupling is a consequence of the strong coupling between the molar fractions and the temperature in the free-flow boundary layer at the interface induced by the additional term $\sum_{i \in \mathcal{C}}-\zeta^{g} h_{i}^{g}\left(d_{\mathrm{t}}+d^{g}\right) \nabla c_{i}$ in the free-flow energy equation (see equation (8)).

In addition to Robin type boundary conditions, we would like to fix also the gas pressure at the interface since its variation in the free-flow domain induced by the coupling is very small. This requires to free the total molar flow rate at the interface since otherwise the gas pressure cannot be prescribed together with the Robin boundary conditions for all components $i \in \mathcal{C}$. This is implemented using the total molar flow rate correction defined at the interface on the free-flow side as additional unknown denoted by $\delta_{\mathbf{v}}^{k}$ and oriented outward to the free-flow domain. This correction is induced by the coupling with the porous medium with prescribed gas pressure $p^{g, k}$ at the interface at iteration $k$ of the domain decomposition algorithm. As can be seen from the last equation of (12), $\delta_{\mathbf{v}}^{k}$ tends to zero at convergence of the domain decomposition algorithm to a fixed point solution. Alternatively, we could keep the gas pressure free at the interface for the Darcy flow subproblem and impose the normal component of the normal stress at the interface for the RANS flow subproblem (12) rather than the total flow rate. Since the gas pressure exhibits small variations, fixing the gas pressure for the Darcy flow subproblem is preferred.

Thus, the porous-medium subproblem solves for the phase pressures $p^{\alpha, k}, \alpha \in \mathcal{P}$, the fugacity vector $\mathbf{f}^{k}$, the temperature $T_{\mathrm{pm}}^{k}$ in $\Omega_{\mathrm{pm}}$ and at the interface $\Gamma$ together with the normal velocity 
correction $\delta_{\mathbf{v}}^{k}$ at the interface $\Gamma$ such that

$$
\begin{aligned}
& \frac{\phi}{\Delta t}\left(\mathrm{n}_{i}^{k}-\mathrm{n}_{i}^{n-1}\right)+\nabla \cdot \mathbf{v}_{i}^{k}=0 \quad \text { in } \Omega_{\mathrm{pm}}, \quad i \in \mathcal{C}, \\
& \frac{1}{\Delta t}\left(E^{k}-E^{n-1}\right)+\nabla \cdot \mathbf{v}_{\mathrm{e}}^{k}=\mathrm{g}_{\mathrm{e}} \text { in } \Omega_{\mathrm{pm}}, \\
& \sum_{i \in \mathcal{C}} c_{i}^{\alpha, k}=1, \quad \text { in } \Omega_{\mathrm{pm}}, \quad \alpha \in \mathcal{P}, \\
& p^{g, k}=\varphi^{k} \quad \text { on } \Gamma, \\
& L c_{i}^{g, k}-\mathbf{v}_{i}^{k} \cdot \mathbf{n}_{\mathrm{pm}}-c_{i}^{g, k} \delta_{\mathbf{v}}^{k}=\varphi_{i}^{k} \quad \text { on } \Gamma, \quad i \in \mathcal{C}, \\
& \sum_{i \in \mathcal{C}} M_{i} c_{i}^{g, k}+N T_{\mathrm{pm}}^{k}-\mathbf{v}_{\mathrm{e}}^{k} \cdot \mathbf{n}_{\mathrm{pm}}-h_{\mathrm{pm}}^{g, k} \delta_{\mathbf{v}}^{k}=\varphi_{\mathrm{e}}^{k} \quad \text { on } \Gamma \text {, } \\
& \sum_{i \in \mathcal{C}} c_{i}^{\alpha, k}=1, \quad \text { on } \Gamma, \quad \alpha \in \mathcal{P},
\end{aligned}
$$

with $h_{\mathrm{pm}}^{g, k}=h^{g}\left(p^{g, k}, \mathbf{c}^{g, k}, T_{\mathrm{pm}}^{k}\right)$ and where the right hand sides

$$
\begin{aligned}
\varphi^{k} & =\mathbf{n}_{\mathrm{ff}} \cdot \mathbb{T}^{k-1} \mathbf{n}_{\mathrm{ff}} \\
\varphi_{i}^{k} & =L c_{i}^{k-1}-\mathbf{w}_{i}^{k-1} \cdot \mathbf{n}_{\mathrm{pm}}, \\
\varphi_{\mathrm{e}}^{k} & =\sum_{i \in \mathcal{C}} M_{i} c_{i}^{k-1}+N T_{\mathrm{ff}}^{k-1}-\mathbf{w}_{\mathrm{e}}^{k-1} \cdot \mathbf{n}_{\mathrm{pm}}
\end{aligned} \quad i \in \mathcal{C},
$$

are given by the previous domain decomposition iterate in the free-flow domain.

\subsection{RANS flow with Dirichlet boundary condition}

Given the normal velocity correction $\delta_{\mathbf{v}}^{k}$ at the interface $\Gamma$, the pressure $p^{k}$ and the gas velocity $\mathbf{u}^{k}$ in the free-flow domain are updated by solving the RANS flow with a Dirichlet boundary condition for the velocity at the interface $\Gamma$ :

$$
\begin{aligned}
\nabla \cdot \mathbb{T}^{k} & =\rho_{\mathrm{ff}}^{g, k} \mathbf{g}, & & \text { in } \Omega_{\mathrm{ff}}, \\
\nabla \cdot\left(\zeta_{\mathrm{ff}}^{g, k} \mathbf{u}^{k}\right) & =0, & & \text { in } \Omega_{\mathrm{ff}}, \\
\zeta_{\mathrm{ff}}^{g, k} \mathbf{u}^{k} & =\zeta_{\mathrm{ff}}^{g, k-1} \mathbf{u}^{k-1}+\delta_{\mathbf{v}}^{k} \mathbf{n}_{\mathrm{ff}}, & & \text { on } \Gamma .
\end{aligned}
$$

In (12), the stress tensor is defined by

$$
\mathbb{T}^{k}=\rho_{\mathrm{ff}}^{g, k} \mathbf{u}^{k} \otimes \mathbf{u}^{k}-\left(\mu_{\mathrm{t}}^{k}+\mu_{\mathrm{ff}}^{g, k}\right)\left(\nabla \mathbf{u}^{k}+\nabla^{\mathrm{t}} \mathbf{u}^{k}\right)+p^{k} \mathbb{I},
$$

with the turbulent viscosity $\mu_{\mathrm{t}}^{k}$ given by the turbulent model which also provides the turbulent diffusivity $d_{\mathrm{t}}^{k}$ and thermal conductivity $\lambda_{\mathrm{t}}^{k}$ that will be used in the following transport subproblem. Note also that, in (12), the gas mass and molar densities are computed using the gas molar fractions and the temperature in the free-flow domain at the previous domain decomposition iterate as follows:

$$
\rho_{\mathrm{ff}}^{g, k}=\rho^{g}\left(p^{k}, T_{\mathrm{ff}}^{k-1}, \mathbf{c}^{k-1}\right), \quad \zeta_{\mathrm{ff}}^{g, k}=\zeta^{g}\left(p^{k}, T_{\mathrm{ff}}^{k-1}, \mathbf{c}^{k-1}\right), \quad \mu_{\mathrm{ff}}^{g, k}=\mu^{g}\left(p^{k}, T_{\mathrm{ff}}^{k-1}, \mathbf{c}^{k-1}\right) .
$$

\subsection{Transport problem with Robin boundary conditions}

Using the gas total molar flow rate $\zeta_{\mathrm{ff}}^{g, k} \mathbf{u}^{k}$ and pressure $p^{k}$ computed at the previous RANS flow step, the molar fractions $\mathbf{c}^{k}$ and the temperature $T_{\mathrm{ff}}^{k}$ are updated solving the transport model in 
the free-flow domain. Robin boundary conditions are imposed at the interface $\Gamma$. They are defined, for the molar conservation of each component $i \in \mathcal{C}$ (resp. of the energy), as a linear combination between the continuity equation of the gas molar fraction $c_{i}$ (resp. the temperature) and the continuity equation of the molar flux $\mathbf{w}_{i} \cdot \mathbf{n}_{\mathrm{ff}}$ (resp. the energy flux $\mathbf{w}_{e} \cdot \mathbf{n}_{\mathrm{ff}}$ ). The coefficients $P$ and $Q$ of these linear combinations in (14) will be computed in order to provide a good low frequency diagonal approximation of the Dirichlet to Neumann operators of respectively a Richards equation for the liquid pressure and of an energy conservation equation for the temperature in the porous-medium domain. It leads to the following transport subproblem:

$$
\begin{aligned}
\nabla \cdot \mathbf{w}_{i}^{k}=0, & \text { in } \Omega_{\mathrm{ff}}, \quad i \in \mathcal{C}, \\
\nabla \cdot \mathbf{w}_{\mathrm{e}}^{k}=0, & \text { in } \Omega_{\mathrm{ff}}, \\
P c_{i}^{k}-\mathbf{w}_{i}^{k} \cdot \mathbf{n}_{\mathrm{ff}}=\psi_{i}^{k} & \text { on } \Gamma, \quad i \in \mathcal{C}, \\
Q T_{\mathrm{ff}}^{k}-\mathbf{w}_{\mathrm{e}}^{k} \cdot \mathbf{n}_{\mathrm{ff}}=\psi_{\mathrm{e}}^{k} & \text { on } \Gamma,
\end{aligned}
$$

where $\mathbf{w}_{i}^{k}=\zeta_{\mathrm{ff}}^{g, k}\left(c_{i}^{k} \mathbf{u}^{k}-\left(d_{\mathrm{t}}^{k}+d^{g, k}\right) \nabla c_{i}^{k}\right)$ is the gas molar flow rate of the component $i \in \mathcal{C}$ using the same definition of the molar density $\zeta_{\mathrm{ff}}^{g, k}$ as in (13), and $\mathbf{w}_{\mathrm{e}}^{k}=\sum_{i \in \mathcal{C}} h_{i}^{g}\left(p^{k}, T_{\mathrm{ff}}^{k}\right) \mathbf{w}_{i}^{k}-\left(\lambda_{\mathrm{t}}^{k}+\lambda^{g, k}\right) \nabla T_{\mathrm{ff}}^{k}$ is the energy flow rate. In (14), the right hand sides are defined by

$$
\begin{aligned}
& \psi_{i}^{k}=P c_{i}^{g, k}-\mathbf{v}_{i}^{k} \cdot \mathbf{n}_{\mathrm{ff}}, \\
& \psi_{\mathrm{e}}^{k}=Q T_{\mathrm{pm}}^{k}-\mathbf{v}_{\mathrm{e}}^{k} \cdot \mathbf{n}_{\mathrm{ff}} .
\end{aligned}
$$

The domain decomposition algorithm is iterated until the following stopping criterion at the interface $\Gamma$ is satisfied for a given tolerance $\varepsilon$ :

$$
\frac{\sum_{i \in \mathcal{C}}\left\|c_{i}^{g, k}-c_{i}^{k}\right\|}{\sum_{i \in \mathcal{C}}\left\|c_{i}^{k}\right\|}+\frac{\left\|T_{\mathrm{pm}}^{k}-T_{\mathrm{ff}}^{k}\right\|}{\left\|T_{\mathrm{ff}}^{k}\right\|}+\frac{\sum_{i \in \mathcal{C}}\left\|\left(\mathbf{v}_{i}^{k}-\mathbf{w}_{i}^{k}\right) \cdot \mathbf{n}_{\mathrm{ff}}\right\|}{\sum_{i \in \mathcal{C}}\left\|\mathbf{w}_{i}^{k} \cdot \mathbf{n}_{\mathrm{ff}}\right\|}+\frac{\left\|\left(\mathbf{v}_{\mathrm{e}}^{k}-\mathbf{w}_{\mathrm{e}}^{k}\right) \cdot \mathbf{n}_{\mathrm{ff}}\right\|}{\left\|\mathbf{w}_{\mathrm{e}}^{k} \cdot \mathbf{n}_{\mathrm{ff}}\right\|}+\frac{\left\|\delta_{\mathbf{v}}^{k}\right\|}{\left\|\zeta_{\mathrm{ff}}^{g, k} \mathbf{u}^{k} \cdot \mathbf{n}_{\mathrm{ff}}\right\|}<\varepsilon,
$$

where $\|\cdot\|$ is a functional norm on $\Gamma$.

Let us show that any fixed point solution of our DDM algorithm satisfies the original physical transmission conditions (9)-(10) at the interface. Gathering the fixed point equations at the interface $\Gamma$ derived from (11)-(12)-(14) by dropping the iteration count $k$, we obtain that

$$
\begin{aligned}
& p^{g}=\mathbf{n}_{\mathrm{ff}} \cdot \mathbb{T} \mathbf{n}_{\mathrm{ff}}, \\
& \delta_{\mathbf{v}} \mathbf{n}_{\mathrm{ff}}=\zeta_{\mathrm{ff}}^{g} \mathbf{u}-\zeta_{\mathrm{ff}}^{g} \mathbf{u}, \\
& P\left(c_{i}^{g}-c_{i}\right)+\left(\mathbf{v}_{i}-\mathbf{w}_{i}\right) \cdot \mathbf{n}_{\mathrm{pm}}=0, i \in \mathcal{C}, \\
& L\left(c_{i}^{g}-c_{i}\right)-\left(\mathbf{v}_{i}-\mathbf{w}_{i}\right) \cdot \mathbf{n}_{\mathrm{pm}}=c_{i}^{g} \delta_{\mathbf{v}}, i \in \mathcal{C}, \\
& Q\left(T_{\mathrm{pm}}-T_{\mathrm{ff}}\right)+\left(\mathbf{v}_{\mathrm{e}}-\mathbf{w}_{\mathrm{e}}\right) \cdot \mathbf{n}_{\mathrm{pm}}=0, \\
& \sum_{i \in \mathcal{C}} M_{i}\left(c_{i}^{g}-c_{i}\right)+N\left(T_{\mathrm{pm}}-T_{\mathrm{ff}}\right)-\left(\mathbf{v}_{\mathrm{e}}-\mathbf{w}_{\mathrm{e}}\right) \cdot \mathbf{n}_{\mathrm{pm}}=h_{\mathrm{pm}}^{g} \delta_{\mathbf{v}}, \\
& \sum_{i \in \mathcal{C}} c_{i}^{\alpha}=1, \alpha \in \mathcal{P} .
\end{aligned}
$$

The second equation implies that $\delta_{\mathbf{v}}=0$. Assuming that $L+P \neq 0$, the third and fourth equations provide that $c_{i}^{g}=c_{i}$ and $\mathbf{v}_{i} \cdot \mathbf{n}_{\mathrm{pm}}=\mathbf{w}_{i} \cdot \mathbf{n}_{\mathrm{pm}}$ for all $i \in \mathcal{C}$. Then, assuming that $N+Q \neq 0$, the fifth and sixth equations imply that $T_{\mathrm{pm}}=T_{\mathrm{ff}}$ and $\mathbf{v}_{\mathrm{e}} \cdot \mathbf{n}_{\mathrm{pm}}=\mathbf{w}_{\mathrm{e}} \cdot \mathbf{n}_{\mathrm{pm}}$. Our construction of the Robin coefficients detailed in the next section guarantees that the coefficients $L, P, N$ and $Q$ are strictly positive. It results that a fixed point solution of the DDM algorithm satisfies the transmission conditions (9)-(10) and hence is a solution of the fully coupled model. 


\section{Computation of the Robin coefficients}

The computation of the Robin coefficients $L, M_{i}, i \in \mathcal{C}$ and $N$ for the Darcy problem (11) and $P$ and $Q$ for the free-flow transport problem (14) is essential to obtain a robust and efficient convergence of the domain decomposition algorithm.

For a scalar linear Partial Differential Equation (PDE) such as a diffusion or a convection diffusion equation on both subdomains, optimized Schwarz methods have been designed. They compute the Robin coefficients in both subdomains simultaneously in order to optimize the convergence rate of the domain decomposition algorithm. These computations assume that the coefficients of the PDEs are constant, either the same in both subdomains [24], [25] or discontinuous at the interface [26].

The extension to nonlinear problems is based on a linear approximation of the PDEs and a freeze of their coefficients at each point of the interface [27]. For systems of PDEs, optimized Robin coefficients are much more difficult to compute. Also, in our case, the turbulent boundary layer plays a major role in the evaporation process and requires to take into account the variable coefficients of the free-flow molar and energy transport problem more accurately than by freezing their values at the interface. This motivates the use of a simpler approach based on diagonal low frequency approximations of the Dirichlet to Neumann (DtN) operator of the neighbouring subdomain problem as explained below.

The DtN operator condensates the neighboring subdomain problem at the interface and is known to define an optimal boundary condition in the sense that, for a linear neighboring subdomain problem, it provides the convergence of the domain decomposition algorithm in two iterations only (see [28]). On the other hand, this operator is dense and expensive to compute exactly. This is why sparse local approximations are built based on differential operators along the interface. The Robin boundary condition corresponds to a zeroth-order differential operator defined as a function along the interface or in other words as a diagonal approximation of the DtN operator. It is called a low frequency approximation because it is, in a sense that is specified below, an exact approximation of the DtN operator on constant modes along the interface. Both constructions of $L, M_{i}, i \in \mathcal{C}, N$, and of $P$ and $Q$ share the same methodology: (i) simplify and linearize the neighboring subdomain problem and (ii) compute a diagonal approximation which is exact on constant modes along the interface. They differ in the way the simplified and linearized models are defined. For $L, M_{i}, i \in \mathcal{C}, N$, the neighboring subdomain problem is the transport free-flow model coupling the molar fractions and the temperature. Its linearization around the free-flow uncoupled solution provides a good approximation of the free-flow transport model. It allows to keep the coupling between the molar fractions and the temperature as well as the variable coefficients which both play a major role in the free-flow domain boundary layer at the interface. It results that the Robin coefficients $L, M_{i}, i \in \mathcal{C}, N$ provide a good approximation of the turbulent conduction and diffusion processes in the free-flow boundary layer. For $P$ and $Q$, the neighboring problem is the highly nonlinear nonisothermal compositional liquid gas Darcy flow. Given its complexity, a rather rough approximation is used based on a the Richards equation for $P$ and the energy equation for $Q$. These simplified models are known to provide a good approximation of the liquid saturation (or liquid pressure) and of the temperature in the porous medium. Both scalar equations are decoupled and linearized and their coefficients are freezed at the interface. Also, the domain is considered unbounded in the normal direction leading to the so-called DtN zeroth-order Taylor approximation which suffices to capture a good order of magnitude of the Robin coefficients $P$ and $Q$ capturing their variation with respect to the time step size and to the relative humidity (see the left Figure 11). Combined with the more accurate approximation of the DtN defined for the coefficients $L, M_{i}, i \in \mathcal{C}, N$, it is shown in the numerical section to provide a good convergence of the domain decomposition algorithm for a wide range of parameters. We now detail both constructions in the following subsections. 


\subsection{Computation of $L, M_{i}, i \in \mathcal{C}$ and $N$}

The diagonal low frequency approximations of the Dirichlet to Neumann operators in the free-flow domain are related to the so-called convective molar and energy transfer coefficients. Their computation can be based on the uncoupled solution of the transport problem in the free-flow domain or alternatively on the coupled solution. Since the perturbation induced by the coupling with the porous-medium domain is small in the free-flow domain, both computations lead to similar results. In our case, it is more convenient to compute the Robin coefficients from the uncoupled solution since the boundary conditions on $\partial \Omega_{\mathrm{ff}} \backslash \Gamma$ and consequently the uncoupled solution are fixed in the numerical experiments of section 5 .

Let us denote by $\left(\mathbf{u}^{0}, p^{0}, \mathbf{c}^{0}, T_{\mathrm{ff}}^{0}\right)$ the solution of the uncoupled RANS flow and transport model (4)-(5)-(7)-(8) in the free-flow domain obtained by considering vanishing molar and energy normal fluxes at the interface $\Gamma$. Let us denote the uncoupled molar flow rates of the components by $i \in \mathcal{C}$

$$
\mathbf{w}_{i}^{0}=\zeta^{g}\left(p^{0}, T_{\mathrm{ff}}^{0}, \mathbf{c}^{0}\right)\left(c_{i}^{0} \mathbf{u}^{0}-\left(d_{\mathrm{t}}^{0}+d^{g, 0}\right) \nabla c_{i}^{0}\right),
$$

and the uncoupled energy flow rate by

$$
\mathbf{w}_{\mathrm{e}}^{0}=\sum_{i \in \mathcal{C}} h_{i}^{g}\left(p^{0}, T_{\mathrm{ff}}^{0}\right) \mathbf{w}_{i}^{0}-\left(\lambda_{\mathrm{t}}^{0}+\lambda^{g, 0}\right) \nabla T_{\mathrm{ff}}^{0} .
$$

By definition they are such that $\left.\mathbf{w}_{i}^{0} \cdot \mathbf{n}_{\mathrm{ff}}\right|_{\Gamma}=0$ and $\left.\mathbf{w}_{\mathrm{e}}^{0} \cdot \mathbf{n}_{\mathrm{ff}}\right|_{\Gamma}=0$.

The mean uncoupled pressure is denoted by $\left\langle p^{0}\right\rangle=\frac{1}{\int_{\Omega_{\mathrm{ff}}} d \mathbf{x}} \int_{\Omega_{\mathrm{ff}}} p^{0}(\mathbf{x}) d \mathbf{x}$ and the mean uncoupled temperature by $\left\langle T_{\mathrm{ff}}^{0}\right\rangle=\frac{1}{\int_{\Omega_{\mathrm{ff}}} d \mathbf{x}} \int_{\Omega_{\mathrm{ff}}} T_{\mathrm{ff}}^{0}(\mathbf{x}) d \mathbf{x}$. Let us also denote by $d_{\mathrm{t}}^{0}$ and $\lambda_{\mathrm{t}}^{0}$ the uncoupled turbulent diffusivity and thermal conductivity and by $d^{g, 0}$ and $\lambda^{g, 0}$ the uncoupled gas Fickian diffusion and standard thermal conductivity.

The Robin coefficients $L, M_{i}, i, j \in \mathcal{C}$ and $N$ are computed from a linear approximation of the transport equations around the uncoupled solution $\mathbf{u}^{0}, p^{0}, \mathbf{c}^{0}, T_{\mathrm{ff}}^{0}$. For the coefficient $L$, a full matrix $L_{i, j}$ with $i, j \in \mathcal{C}$ is first considered and shown to reduce to $L \delta_{i, j}$ in the usual case. Let us define the solutions $\delta \mathbf{c}$ and $\delta T$ of the following linearized transport equations in the free-flow domain with the Dirichlet boundary conditions $\delta \mathbf{c}_{\Gamma}=\left(\delta c_{i, \Gamma}\right)_{i \in \mathcal{C}}$ and $\delta T_{\Gamma}$ at the interface $\Gamma$ :

$$
\begin{aligned}
& \nabla \cdot \delta \mathbf{w}_{i}=0, \quad \text { in } \Omega_{\mathrm{ff}}, \quad i \in \mathcal{C}, \\
& \nabla \cdot \delta \mathbf{w}_{\mathrm{e}}=0, \quad \text { in } \Omega_{\mathrm{ff}} \text {, } \\
& \delta c_{i}=\delta c_{i, \Gamma} \quad \text { on } \Gamma, \quad i \in \mathcal{C}, \\
& \delta T=\delta T_{\Gamma} \quad \text { on } \Gamma \text {, }
\end{aligned}
$$

with homogeneous boundary conditions at $\partial \Omega_{\mathrm{ff}} \backslash \Gamma$ and with

$$
\begin{array}{ll}
\delta \mathbf{w}_{i}=\zeta^{g}\left(p^{0}, T_{\mathrm{ff}}^{0}, \mathbf{c}^{0}\right)\left(\delta c_{i} \mathbf{u}^{0}-\left(d_{\mathrm{t}}^{0}+d^{g, 0}\right) \nabla \delta c_{i}\right), & i \in \mathcal{C}, \\
\delta \mathbf{w}_{\mathrm{e}}=\sum_{i \in \mathcal{C}}\left(h_{i}^{g}\left(p^{0}, T_{\mathrm{ff}}^{0}\right) \delta \mathbf{w}_{i}+\frac{\partial h_{i}^{g}}{\partial T}\left(\left\langle p^{0}\right\rangle,\left\langle T_{\mathrm{ff}}^{0}\right\rangle\right) \delta T \mathbf{w}_{i}^{0}\right)-\left(\lambda_{\mathrm{t}}^{0}+\lambda^{g, 0}\right) \nabla \delta T . &
\end{array}
$$

The choice of $\frac{\partial h_{i}^{g}}{\partial T}\left(\left\langle p^{0}\right\rangle,\left\langle T_{\mathrm{ff}}^{0}\right\rangle\right)$ rather than $\frac{\partial h_{i}^{g}}{\partial T}\left(p^{0}, T_{\mathrm{ff}}^{0}\right)$ is motivated by the maximum principle for the solution of equation (20) which is obtained only for a divergence free velocity.

The Dirichlet to Neumann operators related to the linearized transport equations (16) are defined from these solutions $\delta \mathbf{c}$ and $\delta T$ by

$$
\begin{aligned}
& \left(\delta \mathbf{c}_{\Gamma}, \delta T_{\Gamma}\right) \rightarrow \operatorname{DtN}_{i}\left(\delta \mathbf{c}_{\Gamma}, \delta T_{\Gamma}\right)=-\left.\delta \mathbf{w}_{i} \cdot \mathbf{n}_{\mathrm{ff}}\right|_{\Gamma}, \quad i \in \mathcal{C} \\
& \left(\delta \mathbf{c}_{\Gamma}, \delta T_{\Gamma}\right) \rightarrow \operatorname{DtN}_{\mathrm{e}}\left(\delta \mathbf{c}_{\Gamma}, \delta T_{\Gamma}\right)=-\left.\delta \mathbf{w}_{\mathrm{e}} \cdot \mathbf{n}_{\mathrm{ff}}\right|_{\Gamma} .
\end{aligned}
$$


The Robin coefficients are built from low frequency diagonal approximations of these Dirichlet to Neumann operators computed in order to be exact on the constant basis functions for $\delta \mathbf{c}_{\Gamma}$ and $\delta T_{\Gamma}$ along the interface $\Gamma$. Hence, for $i \in \mathcal{C}$, let us define $\delta \mathbf{e}^{(i)}$ such that $\delta e_{j}^{(i)}=\delta_{i, j}, j \in \mathcal{C}$. Then we set

$$
\begin{aligned}
L_{i, j} & =\operatorname{DtN}_{i}\left(\delta \mathbf{e}^{(j)}, 0\right), \quad i, j \in \mathcal{C}, \\
M_{i} & =\operatorname{DtN}_{\mathrm{e}}\left(\delta \mathbf{e}^{(i)}, 0\right), \quad i \in \mathcal{C}, \\
N & =\operatorname{DtN}_{\mathrm{e}}(\mathbf{0}, 1) .
\end{aligned}
$$

Provided that, as usual, the boundary conditions on $\partial \Omega_{\mathrm{ff}} \backslash \Gamma$ for the molar transport equations do not couple the components and are of the same type for all components $i \in \mathcal{C}$, it is clear that $L_{i, j}=L \delta_{i, j}$ for all $i, j \in \mathcal{C}$ where $L$ is defined by the following function along the interface $\Gamma$ :

$$
L(\mathbf{x})=-\zeta^{g}\left(p^{0}, T_{\mathrm{ff}}^{0}, \mathbf{c}^{0}\right)\left(d_{\mathrm{t}}^{0}+d^{g, 0}\right) \nabla \delta c_{1} \cdot \mathbf{n}_{\mathrm{ff}}(\mathbf{x}), \mathbf{x} \in \Gamma,
$$

with $\delta c_{1}$ solution of the following scalar convection diffusion equation:

$$
\begin{array}{rr}
\nabla \cdot\left(\zeta^{g}\left(p^{0}, T_{\mathrm{ff}}^{0}, \mathbf{c}^{0}\right)\left(\delta c_{1} \mathbf{u}^{0}-\left(d_{\mathrm{t}}^{0}+d^{g, 0}\right) \nabla \delta c_{1}\right)\right)=0, & \text { in } \Omega_{\mathrm{ff}}, \\
\delta c_{1}=1 & \text { on } \Gamma .
\end{array}
$$

Similarly, the Robin coefficient $N$ is defined by the following function along the interface $\Gamma$ :

$$
N(\mathbf{x})=-\left(\lambda_{\mathrm{t}}^{0}+\lambda^{g, 0}\right) \nabla \delta T \cdot \mathbf{n}_{\mathrm{ff}}(\mathbf{x}), \mathbf{x} \in \Gamma,
$$

with $\delta T$ solution of the following scalar convection diffusion equation:

$$
\begin{aligned}
& \nabla \cdot\left(\sum_{i \in \mathcal{C}}\left(\frac{\partial h_{i}^{g}}{\partial T}\left(\left\langle p^{0}\right\rangle,\left\langle T_{\mathrm{ff}}^{0}\right\rangle\right) \delta T \mathbf{w}_{i}^{0}\right)-\left(\lambda_{\mathrm{t}}^{0}+\lambda^{g, 0}\right) \nabla \delta T\right)=0, \quad \text { in } \Omega_{\mathrm{ff}}, \\
& \delta T=1 \quad \text { on } \Gamma .
\end{aligned}
$$

Using the solution $\delta c_{1}$ of equation (18), let us set

$$
\delta \mathbf{w}_{1}=\zeta^{g}\left(p^{0}, T_{\mathrm{ff}}^{0}, \mathbf{c}^{0}\right)\left(\delta c_{1} \mathbf{u}^{0}-\left(d_{\mathrm{t}}^{0}+d^{g, 0}\right) \nabla \delta c_{1}\right) .
$$

The solution $\delta \mathbf{c}^{(i)}, \delta T^{(i)}$ of equations (16) with the Dirichlet boundary conditions $\delta \mathbf{c}=\delta \mathbf{e}^{(i)}, \delta T=0$ on $\Gamma$ is given by $\delta c_{i}^{(i)}=\delta c_{1}$ and $\delta c_{j}^{(i)}=0$ for $j \neq i$ and the solution $\delta T^{(i)}$ of the following convection diffusion equation with a source term and an homogeneous Dirichlet boundary condition on $\Gamma$ :

$$
\begin{aligned}
\nabla \cdot\left(\sum_{j \in \mathcal{C}}\left(\frac{\partial h_{j}^{g}}{\partial T}\left(\left\langle p^{0}\right\rangle,\left\langle T_{\mathrm{ff}}^{0}\right\rangle\right) \delta T^{(i)} \mathbf{w}_{j}^{0}\right)-\left(\lambda_{\mathrm{t}}^{0}+\lambda^{g, 0}\right) \nabla \delta T^{(i)}\right) & =-\nabla \cdot\left(h_{i}^{g}\left(p^{0}, T_{\mathrm{ff}}^{0}\right) \delta \mathbf{w}_{1}\right) & \text { in } \Omega_{\mathrm{ff}}, \\
\delta T^{(i)} & =0 & \text { on } \Gamma,
\end{aligned}
$$

Then, the Robin coefficient $M_{i}, i \in \mathcal{C}$ is defined from the solution $\delta T^{(i)}$ by

$$
M_{i}(\mathbf{x})=\left(-\left(\lambda_{\mathrm{t}}^{0}+\lambda^{g, 0}\right) \nabla \delta T^{(i)}-h_{i}^{g}\left(p^{0}, T_{\mathrm{ff}}^{0}\right) \zeta^{g}\left(p^{0}, T_{\mathrm{ff}}^{0}, \mathbf{c}^{0}\right)\left(d_{\mathrm{t}}^{0}+d^{g, 0}\right) \nabla \delta c_{1}\right) \cdot \mathbf{n}_{\mathrm{ff}}(\mathbf{x}), \mathbf{x} \in \Gamma .
$$

Using that $\nabla \cdot \mathbf{w}_{i}^{0}=0$ for all component $i \in \mathcal{C}$, it results from the maximum principle [29] applied to the convection diffusion equations (18) and (20) that the Robin coefficients $L$ and $N$ are strictly positive which ensures the well-posedness of the porous medium subproblem of subsection 3.1. In practice, these scalar convection diffusion equations are solved numerically using the same discretization than the one used for the transport equations in the free-flow domain. 
Remark 1. Assuming that the uncoupled temperature $T_{\mathrm{ff}}^{0}$ is constant and neglecting the uncoupled pressure $p^{0}$ variations, the right hand side in equation (21) vanishes and hence $\delta T^{(i)}=0$. It results, in that case, that the following relation between the Robin coefficients $M_{i}$ and $L$ holds for all $i \in \mathcal{C}$ :

$$
M_{i}(\mathbf{x})=h_{i}^{g}\left(p^{0}, T_{\mathrm{ff}}^{0}\right) L(\mathbf{x}) .
$$

Remark 2. Let us consider the case of an air (a) and water (w) system, and let us assume that $c_{w}^{0}<<c_{a}^{0}$, that $\mathbf{c}^{0}$ is constant and that $h_{a}^{g}(T)=C_{\mathrm{p}, a}^{g} m_{a} T$ where $C_{\mathrm{p}, a}^{g}$ is the specific heat capacity of pure air and $m_{a}$ the air molar mass. It results that the following approximation

$$
\sum_{i \in \mathcal{C}}\left(\frac{\partial h_{i}^{g}}{\partial T}\left(\left\langle p^{0}\right\rangle,\left\langle T_{\mathrm{ff}}^{0}\right\rangle\right) \delta T \mathbf{w}_{i}^{0}\right) \simeq C_{\mathrm{p}, a}^{g} \zeta^{g} m_{a} \delta T \mathbf{u}^{0},
$$

can be made in equation (20). Let us also assume that the following typical relation holds between the diffusivity and the thermal conductivity:

$$
\left(\lambda_{\mathrm{t}}^{0}+\lambda^{g, 0}\right) \simeq C_{\mathrm{p}, a}^{g} \zeta^{g} m_{a} S_{\mathrm{c}}\left(d_{\mathrm{t}}^{0}+d^{g, 0}\right),
$$

where $S_{\mathrm{c}}$ is the Schmidt number. Assuming $S_{\mathrm{c}}=1$, it results from equations (18), (20) and from the definition (17) of $L$ and (19) of $N$ that the following approximate relation between the Robin coefficients $L$ and $N$ holds

$$
N(\mathbf{x}) \simeq C_{\mathrm{p}, a}^{g} m_{a} L(\mathbf{x})
$$

\subsection{Computation of $P$ and $Q$}

Our strategy to compute the Robin coefficients $P$ and $Q$ is based on the Richards equation coupled with the energy conservation equation. This simplified system provides a good approximation of the liquid pressure and of the temperature. Then, after time integration using an Euler implicit scheme, these equations are linearized and their coefficients are freezed at each point of the interface $\Gamma$ leading to a constant coefficient linear system of two PDEs with two unknowns. A zeroth-order Taylor diagonal approximation of the Dirichlet to Neumann operator related to this PDE system leads to a 2 by 2 matrix (see [28]) which is evaluated on the current solution obtained at each point of the interface. To simplify the computations, the Richards and energy conservation equations will be decoupled leading to a diagonal 2 by 2 matrix defining precisely the coefficients $P$ and $Q$ with simple analytical formulae evaluated at each point of the interface $\Gamma$. In practice, this decoupled approach suffices to obtain a good convergence as will be exhibited in the numerical section 5 .

The simplified system coupling the Richards and energy conservation equations is obtained by neglecting the dissolution of the gaseous component in the liquid phase and by neglecting the variation of the gas pressure which is approximated by the reference pressure denoted by $p_{\text {ref }}^{g}$ typically given by the outflow pressure in the free-flow domain (see [30]).

Let us define the pure water molar fractions $\overline{\mathbf{c}}^{\ell}$ in the liquid phase by $\bar{c}_{w}^{\ell}=1$ and $\bar{c}_{j}^{\ell}=0$ for $j \in \mathcal{C} \backslash\{w\}$. The molar fractions $\overline{\mathbf{c}}^{g}$ in the gas phase at thermodynamical equilibrium with $\overline{\mathbf{c}}^{\ell}$ are given for each components $i \in \mathcal{C}$ by

$$
\bar{c}_{i}^{g}\left(p^{\ell}, T_{\mathrm{pm}}\right)=c_{i}^{g}\left(p_{\mathrm{ref}}^{g}, T_{\mathrm{pm}}, f^{\ell}\left(p^{\ell}, T_{\mathrm{pm}}, \overline{\mathbf{c}}^{\ell}\right)\right) .
$$

Let us define $\bar{s}^{l}\left(\mathbf{x}, p^{l}\right)=s^{l}\left(\mathbf{x}, p_{\text {ref }}^{g}-p^{l}\right)$ and let us denote the thermodynamical laws as functions of $p^{\ell}$ and $T_{\mathrm{pm}}$ by $\bar{\zeta}^{\alpha}\left(p^{\ell}, T_{\mathrm{pm}}\right), \bar{\rho}^{\alpha}\left(p^{\ell}, T_{\mathrm{pm}}\right), \bar{e}^{\alpha}\left(p^{\ell}, T_{\mathrm{pm}}\right), \bar{h}^{\alpha}\left(p^{\ell}, T_{\mathrm{pm}}\right)$, and $\bar{\mu}^{\alpha}\left(p^{\ell}, T_{\mathrm{pm}}\right)$ for $\alpha \in \mathcal{P}$. Finally 
let us set

$$
\begin{array}{rlr}
\overline{\mathrm{n}}_{w}\left(\mathbf{x}, p^{\ell}, T_{\mathrm{pm}}\right) & =\bar{\zeta}^{\ell} \bar{s}^{\ell}+\bar{\zeta}^{g}\left(1-\bar{s}^{\ell}\right) \bar{c}_{w}^{g}, & M^{\ell}\left(\mathbf{x}, p^{\ell}, T_{\mathrm{pm}}\right)=\frac{\bar{\zeta}^{\ell}}{\bar{\mu}^{\ell}} k_{\mathrm{r}}^{\ell}\left(\mathbf{x}, \bar{s}^{\ell}\right), \\
\bar{E}\left(\mathbf{x}, p^{\ell}, T_{\mathrm{pm}}\right) & =\phi\left(\bar{s}^{\ell} \bar{e}^{\ell}+\left(1-\bar{s}^{\ell}\right) \bar{e}^{g}\right)+(1-\phi) \zeta^{r} e^{r} . &
\end{array}
$$

The Richards equation with prescribed water molar fraction $c_{w, \Gamma}$ at the interface $\Gamma$ is defined as follows after Euler implicit time integration:

$$
\begin{aligned}
\frac{\phi}{\Delta t}\left(\overline{\mathrm{n}}_{w}-\overline{\mathrm{n}}_{w}^{n-1}\right)+\nabla \cdot \overline{\mathbf{v}}_{w} & =0, & & \text { in } \Omega_{\mathrm{pm}}, \\
\bar{c}_{w}^{g}\left(p^{\ell}, T_{\mathrm{pm}}\right) & =c_{w, \Gamma}, & & \text { on } \Gamma,
\end{aligned}
$$

where

$$
\overline{\mathbf{v}}_{w}=-M^{\ell} \mathbb{K}\left(\nabla p^{\ell}-\bar{\rho}^{\ell} \mathbf{g}\right)-d_{\mathrm{pm}}^{g}\left(\mathbf{x}, 1-\bar{s}^{\ell}\right) \nabla \bar{c}_{w}^{g}\left(p^{\ell}, T_{\mathrm{pm}}\right) .
$$

The simplified energy conservation equation with prescribed temperature $T_{\Gamma}$ at the interface $\Gamma$ is defined by

$$
\begin{aligned}
\frac{1}{\Delta t}\left(\bar{E}-\bar{E}^{n-1}\right)+\nabla \cdot \overline{\mathbf{v}}_{e} & =0, & & \text { in } \Omega_{\mathrm{pm}}, \\
T_{\mathrm{pm}} & =T_{\Gamma}, & & \text { on } \Gamma
\end{aligned}
$$

where

$$
\overline{\mathbf{v}}_{e}=-M^{\ell} \overline{\mathrm{h}}^{\ell} \mathbb{K}\left(\nabla p^{\ell}-\bar{\rho}^{\ell} \mathbf{g}\right)-\lambda_{\mathrm{pm}}\left(\mathbf{x}, \bar{s}^{\ell}\right) \nabla T_{\mathrm{pm}} .
$$

To compute the Robin coefficient $P$, the Richards equation (23) is linearized with respect to $p^{\ell}$ at fixed temperature $T_{\mathrm{pm}}$ leading to the equation

$$
\eta \delta p^{\ell}-\nabla \cdot\left(\kappa \nabla \delta p^{\ell}-\mathbf{\Psi} \delta p^{\ell}\right)=0, \text { in } \Omega_{\mathrm{pm}},
$$

with the Dirichlet boundary condition

$$
\delta p^{\ell}=\xi \delta c_{w} \text { on } \Gamma
$$

The coefficients are defined by

$$
\xi=\frac{1}{\frac{\partial \bar{c}_{w}^{g}}{\partial p^{\ell}}}, \quad \eta=\frac{\phi}{\Delta t} \frac{\partial \overline{\mathrm{n}}_{w}}{\partial p^{\ell}}, \quad \kappa=M^{\ell} \mathbb{K}+d_{\mathrm{pm}} \frac{\partial \bar{c}_{w}^{g}}{\partial p^{\ell}} \mathbb{I}, \quad \Psi=-\frac{\partial M^{\ell}}{\partial p^{\ell}} \mathbb{K} \nabla p^{\ell}+\frac{\partial M^{\ell} \bar{\rho}^{\ell}}{\partial p^{\ell}} \mathbb{K} \mathbf{g}-\frac{\partial d_{\mathrm{pm}}}{\partial p^{\ell}} \nabla \bar{c}_{w}^{g} .
$$

The Robin coefficient $P$ is obtained using the DtN zeroth-order Taylor approximation [28] of this linear scalar equation with freezed coefficients at each point of the interface $\Gamma$. The values of the coefficients $\xi, \eta, \kappa$ and $\boldsymbol{\Psi}$ are freezed at given position $\mathbf{x} \in \Gamma$, time $t^{n}$, liquid pressure $p^{\ell}\left(\mathbf{x}, t^{n}\right)$, and temperature $T_{\mathrm{pm}}\left(\mathbf{x}, t^{n}\right)$. Then, considering a constant mode along the interface $\Gamma$ as a low frequency approximation, the equation (25) is integrated along the normal direction $\mathbf{x}+n \mathbf{n}_{\mathrm{ff}}$ with $n \in(0,+\infty)$ using the Dirichlet boundary condition (26) obtained with $\delta c_{w}=1$. It is complemented by a boundedness condition when $n$ goes to infinity.

$$
\begin{aligned}
& \eta \delta p^{\ell}(n)-\left(\kappa \mathbf{n}_{\mathrm{ff}} \cdot \mathbf{n}_{\mathrm{ff}}\right) \frac{d^{2} \delta p^{\ell}}{d n^{2}}(n)+\left(\boldsymbol{\Psi} \cdot \mathbf{n}_{\mathrm{ff}}\right) \frac{d \delta p^{\ell}}{d n}(n)=0 \text { on }(0,+\infty), \\
& \delta p^{\ell}(0)=\xi \\
& \lim _{n \rightarrow+\infty}\left|\delta p^{\ell}(n)\right|<+\infty
\end{aligned}
$$

The normal flux at the interface $n=0$ defined by

$$
-\left(\kappa \mathbf{n}_{\mathrm{ff}} \cdot \mathbf{n}_{\mathrm{ff}}\right) \frac{d \delta p^{\ell}}{d n}(0)+\left(\boldsymbol{\Psi} \cdot \mathbf{n}_{\mathrm{ff}}\right) \delta p^{\ell}(0),
$$


is the DtN zeroth-order Taylor approximation and is equal to

$$
P\left(\mathbf{x}, t^{n}\right)=\frac{\xi}{2}\left(\boldsymbol{\Psi} \cdot \mathbf{n}_{\mathrm{ff}}+\sqrt{\left(\Psi \cdot \mathbf{n}_{\mathrm{ff}}\right)^{2}+4 \eta \kappa \mathbf{n}_{\mathrm{ff}} \cdot \mathbf{n}_{\mathrm{ff}}}\right) .
$$

The Robin coefficient $Q$ is computed using the same methodology. We first linearize the energy conservation equation (24) with respect to $T_{\mathrm{pm}}$ at fixed liquid pressure $p^{\ell}$ and freeze its coefficients at each point of the interface $\Gamma$. Then, $Q\left(\mathbf{x}, t^{n}\right)$ is equal to the zeroth-order Taylor approximation of the DtN operator of this constant coefficient linear scalar equation. The same formula as for $P$ is obtained with new coefficients defined by

$$
\xi=1, \quad \eta=\frac{1}{\Delta t} \frac{\partial \bar{E}}{\partial T_{\mathrm{pm}}}, \quad \kappa=\lambda_{\mathrm{pm}}, \quad \boldsymbol{\Psi}=-\frac{\partial M^{\ell} \bar{h}^{\ell}}{\partial T_{\mathrm{pm}}} \mathbb{K} \nabla p^{\ell}+\frac{\partial M^{\ell} \bar{h}^{\ell} \bar{\rho}^{\ell}}{\partial T_{\mathrm{pm}}} \mathbb{K} \mathbf{g} .
$$

Let us remark that the Robin coefficients $P$ and $Q$ are strictly positive which ensures the well-posedness of the transport subproblem of subsection 3.3 .

\section{$5 \quad$ Numerical experiments}

In order to assess the efficiency of the domain decomposition algorithm, we consider in the following tests a simple 2D setup with the free-flow vertical domain $\Omega_{\mathrm{ff}}=(0, l) \times\left(0, h_{\mathrm{ff}}\right)$ and the porous-medium vertical domain $\Omega_{\mathrm{pm}}=(0, l) \times\left(h_{\mathrm{ff}}, h_{\mathrm{pm}}\right)$ sharing the interface $\Gamma=(0, l) \times\left\{h_{\mathrm{ff}}\right\}$ with $h_{\mathrm{pm}}>h_{\mathrm{ff}}>0$ and $l>0$.

The top boundary of the porous medium is denoted by $\Gamma_{\mathrm{up}}=(0, l) \times\left\{h_{\mathrm{pm}}\right\}$, the output boundary of the free-flow domain is denoted by $\Gamma_{\text {out }}=\{l\} \times\left(0, h_{\mathrm{ff}}\right)$, and the input boundary by $\Gamma_{\mathrm{in}}=\{0\} \times\left(0, h_{\mathrm{ff}}\right)$.

The liquid and gas phases are considered as mixtures of air $(a)$ and water $(w)$ defining the set of components $\mathcal{C}=\{a, w\}$. The liquid molar density is fixed to $\zeta^{\ell}=55555 \mathrm{~mol} \cdot \mathrm{m}^{-3}$ and its viscosity to $\mu^{\ell}=10^{-3} \mathrm{~Pa} \cdot \mathrm{s}$. The gas molar density is given by the perfect gas law

$$
\zeta^{g}=\frac{p^{g}}{\mathrm{RT}} .
$$

where $\mathrm{R}=8.314 \mathrm{~J} \cdot \mathrm{mol}^{-1} \cdot \mathrm{K}^{-1}$ is the ideal gas constant. The gas viscosity $\mu^{g}=1.851 \cdot 10^{-5} \mathrm{~Pa} \cdot \mathrm{s}$ is assumed constant. The mass density of the phase $\alpha \in \mathcal{P}$ is deduced from the molar density by the relation

$$
\rho^{\alpha}\left(p^{\alpha}, T, \mathbf{c}^{\alpha}\right)=\zeta^{\alpha}\left(p^{\alpha}, T, \mathbf{c}^{\alpha}\right) \sum_{i \in \mathcal{C}} c_{i}^{\alpha} m_{i}
$$

where the molar masses of the water component and of the air component are given respectively by $m_{w}=18 \cdot 10^{-3} \mathrm{~kg} \cdot \mathrm{mol}^{-1}$ and by $m_{a}=29 \cdot 10^{-3} \mathrm{~kg} \cdot \mathrm{mol}^{-1}$.

The fugacities of the components in the gas phase are given by Dalton's law for an ideal mixture of perfect gas

$$
f_{i}^{g}=c_{i}^{g} p^{g}, \quad i \in \mathcal{C} .
$$

The fugacities of the components in the liquid phase are given by Henry's law for the dissolution of the air component in the liquid phase

$$
f_{a}^{\ell}=c_{a}^{\ell} H_{a}(T), \quad H_{a}(T)=H_{1}+\frac{T-T_{1}}{T_{2}-T_{1}}\left(H_{2}-H_{1}\right),
$$

with $H_{1}=6 \cdot 10^{9} \mathrm{~Pa}, H_{2}=10^{10} \mathrm{~Pa}, T_{1}=293 \mathrm{~K}, T_{2}=353 \mathrm{~K}$, and by Raoult-Kelvin's law for the water component in the liquid phase

$$
f_{e}^{\ell}=c_{e}^{\ell} p_{\text {sat }}(T) \exp \left(\frac{p^{\ell}-p_{\text {sat }}(T)}{\zeta^{\ell} \mathrm{R} T}\right)
$$


where $p_{\text {sat }}(T)$ is the vapor pressure of the pure water given by the Rankine's formula. The molar fractions $\mathbf{c}^{\alpha}$ as functions of $p^{\alpha}, T, f$ are deduced by inversion of the fugacity equations (1) leading to

$$
\begin{aligned}
c_{w}^{\ell}\left(p^{\ell}, T, \mathbf{f}\right) & =\frac{f_{w}}{p_{\text {sat }}(T)} \exp \left(\frac{p_{\text {sat }}(T)-p^{\ell}}{\zeta^{\ell} \mathrm{R} T}\right), \\
c_{a}^{\ell}\left(p^{\ell}, T, \mathbf{f}\right) & =\frac{f_{a}}{H^{a}(T)}, \\
c_{i}^{g}\left(p^{g}, T, \mathbf{f}\right) & =\frac{f_{i}}{p^{g}}, \quad i \in \mathcal{C} .
\end{aligned}
$$

The liquid molar enthalpy is taken from [31] and the gas molar enthalpy is defined by

$$
h^{g}\left(p^{g}, T_{\mathrm{pm}}, \mathbf{c}^{g}\right)=\sum_{i \in \mathcal{C}} h_{i}^{g}\left(p^{g}, T_{\mathrm{pm}}\right) c_{i}^{g} .
$$

with the enthalpy of each component given in [31] for the water component and by

$$
h_{a}^{g}(T)=C_{\mathrm{p}, a}^{g} m_{a} T
$$

for the air component, where $C_{\mathrm{p}, a}^{g}=1000 \mathrm{~J} \cdot \mathrm{K}^{-1} \cdot \mathrm{kg}^{-1}$ is the specific heat capacity of pure air. In the porous-medium domain, the rock internal energy per unit rock volume is given by $\zeta^{r} e^{r}(T)=2 \cdot 10^{6} \mathrm{~T} \mathrm{~J} \cdot \mathrm{K}^{-1} \cdot \mathrm{m}^{-3}$. The thermal conductivity of the liquid gas rock mixture is considered as constant for the sake of simplicity and fixed to $\lambda_{\mathrm{pm}}=2 \mathrm{~W} \cdot \mathrm{K}^{-1} \cdot \mathrm{m}^{-1}$.

The liquid saturation and the phase relative permeabilities are given by the Van Genuchten laws defined by

$$
s^{\ell}\left(p_{\mathrm{c}}\right)=s_{\mathrm{r}}^{\ell}+\left(1-s_{\mathrm{r}}^{\ell}-s_{\mathrm{r}}^{g}\right)\left(1+\left(p_{\mathrm{r}}^{-1} p_{\mathrm{c}}\right)^{n_{\mathrm{r}}}\right)^{-m_{\mathrm{r}}},
$$

and

$$
\begin{aligned}
& k_{\mathrm{r}}^{\ell}\left(s^{\ell}\right)= \begin{cases}0 & \text { if } s^{\ell}<s_{\mathrm{r}}^{\ell}, \\
\left(1-\left(1-\left(\bar{s}^{\ell}\right)^{1 / m_{r}}\right)^{m_{r}}\right)^{2} \sqrt{\bar{s}^{\ell}} & \text { if } s_{\mathrm{r}}^{\ell} \leq s^{\ell}<1-s_{\mathrm{r}}^{g}, \\
1 & \text { otherwise }\end{cases} \\
& k_{\mathrm{r}}^{g}\left(s^{g}\right)= \begin{cases}0 & \text { if } s^{g}<s_{\mathrm{r}}^{g}, \\
\left(1-\left(\bar{s}^{\ell}\right)^{1 / m_{r}}\right)^{2 m_{r}} \sqrt{1-\bar{s}^{\ell}} & \text { if } s_{\mathrm{r}}^{g} \leq s^{g}<1-s_{\mathrm{r}}^{\ell}, \\
1 & \text { otherwise }\end{cases}
\end{aligned}
$$

with

$$
\bar{s}^{\ell}\left(s^{\ell}\right)=\frac{s^{\ell}-s_{\mathrm{r}}^{\ell}}{1-s_{\mathrm{r}}^{\ell}-s_{\mathrm{r}}^{g}},
$$

and $m_{\mathrm{r}}=1-n_{\mathrm{r}}^{-1}$. The parameters $s_{\mathrm{r}}^{\ell}, s_{\mathrm{r}}^{g}, p_{\mathrm{r}}$ and $n_{\mathrm{r}}$ will be specified for each test case according to the rocktype.

In the free-flow domain, the turbulent viscosity $\mu_{\mathrm{t}}$ used to define the RANS stress tensor (6) is given by the Prandtl algebraic turbulent model as in [5] and computed once and for all from the uncoupled solution in the free-flow model. The turbulent diffusivity

$$
d_{\mathrm{t}}=\frac{\mu_{\mathrm{t}}}{\rho^{g} S_{\mathrm{c}}}
$$

is deduced using the Schmidt number $S_{\mathrm{c}}=1$, and the gas Fickian diffusion coefficient is fixed to $d^{g}=2 \cdot 10^{-5} \mathrm{~m}^{2} \cdot \mathrm{s}^{-1}$. The turbulent thermal conductivity is similarly defined by $\lambda_{\mathrm{t}}=C_{\mathrm{p}, a}^{g} \mu_{\mathrm{t}}$ and the gas thermal conductivity is fixed to $\lambda^{g}=0.026 \mathrm{~W} \cdot \mathrm{m}^{-1} \cdot \mathrm{K}^{-1}$.

Following [5], a Cartesian mesh uniform in the $x$ direction and refined at the interface $\Gamma$ on both sides is used. The Darcy problem (11) and the convection diffusion equations (14) are solved using a 


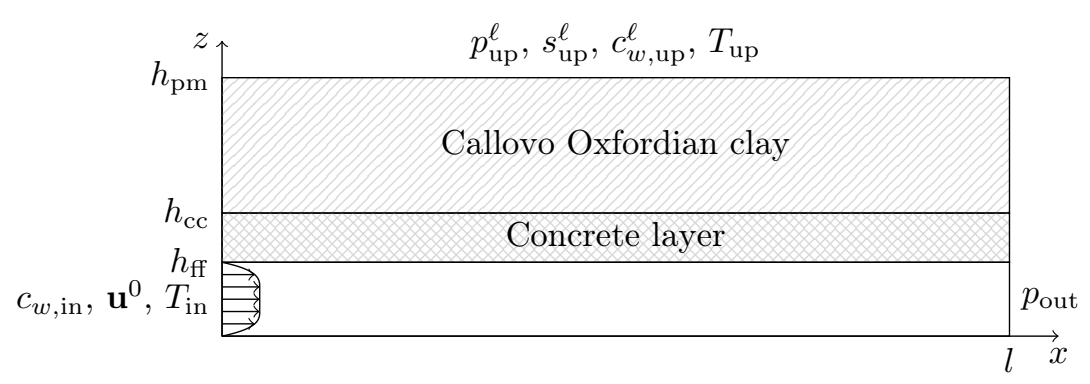

Figure 1 - Setup of the Andra test case 1.

two-point flux approximation scheme given in [32] with additional face unknowns at the interface $\Gamma$ to discretize the boundary conditions. The RANS model in (12) is solved using a staggered Marker And Cell scheme given in [33]. An implicit Euler scheme is used for the time integration using the time stepping

$$
\begin{aligned}
\Delta t^{1} & =\Delta t_{0}, \\
\Delta t^{n} & =\min \left(\rho_{\delta t} \Delta t^{n-1}, \Delta t_{\max }\right), \quad n>1,
\end{aligned}
$$

with an initial time step $\Delta t_{0}$, a growth rate $\rho_{\delta t}$ and a maximum time step $\Delta t_{\max } \geq \Delta t_{0}$. The final simulation time is denoted by $t_{\mathrm{f}}$. The nonlinear systems obtained at each time step and at each domain decomposition iteration are solved using a Newton algorithm for both the Darcy (11) and transport (14) subproblems and a Quasi Newton algorithm described in [5] for the RANS flow subproblem (12). The sizes of the linear systems for the Darcy and transport subproblems amount to 3 conservation equations and unknowns per cell and 3 equations and unknowns per face at the interface $\Gamma$. This reduction is achieved for the Darcy problem thanks to the elimination of the local closure laws. The linear system for the RANS flow Quasi-Newton algorithm couples the two components of the velocity and the pressure. It is based on the uncoupled RANS flow model leading to a linear system independent of time which can be factorized once and for all. The solution of each linear system is computed at each nonlinear solver iteration using the sequential version of the SuperLU direct sparse solver [34], [35].

The first three test cases are simplified two dimensional test cases defined with Andra [5] to simulate the mass and energy exchanges occurring within deep geological radioactive waste disposal at the interface between a geological formation with low permeable porous medium and a ventilated excavated gallery. The data sets are derived from lab experiments and in accordance with the deep disposal center for French radioactive waste project. The fourth test case considers the convective drying of a porous medium with a much larger permeability.

\subsection{Andra test case 1}

For this first Andra test case, we consider the domain defined by $l=100 \mathrm{~m}, h_{\mathrm{pm}}=15 \mathrm{~m}$ and $h_{\mathrm{ff}}=5 \mathrm{~m}$. As exhibited in Figure 1, the porous medium includes a layer of concrete rocktype in the domain $\Omega_{\mathrm{cc}}=(0, l) \times\left(h_{\mathrm{ff}}, h_{\mathrm{cc}}\right)$ with $h_{\mathrm{cc}}=6 \mathrm{~m}$ and a Callovo Oxfordian clay (COx) rocktype in the remaining domain $\Omega_{\mathrm{ox}}=\Omega_{\mathrm{pm}} \backslash \bar{\Omega}_{\mathrm{cc}}$.

The porosity and the isotropic permeability are set according to the rocktype such that

$$
\phi=\left\{\begin{array}{ll}
0.3 & \text { in } \Omega_{\mathrm{cc}}, \\
0.15 & \text { in } \Omega_{\mathrm{ox}},
\end{array} \quad \mathbb{K}= \begin{cases}10^{-18} \mathrm{~m}^{2} & \text { in } \Omega_{\mathrm{cc}} \\
5 \cdot 10^{-20} \mathrm{~m}^{2} & \text { in } \Omega_{\mathrm{ox}}\end{cases}\right.
$$

The Van Genuchten parameters of each rocktype, governing the liquid saturation and the relative 
(a) Liquid saturation

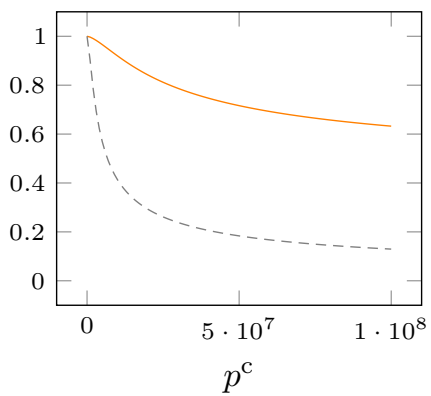

(b) Relative permeability

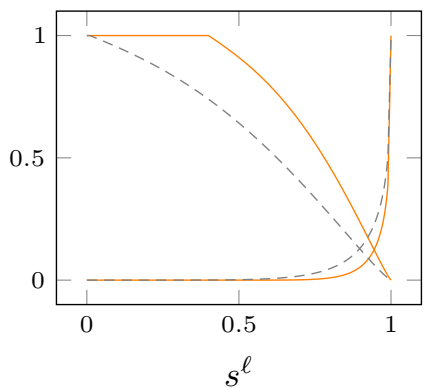

Figure 2 - Liquid saturation function and relative permeabilities of both phases in the Callovo Oxfordian clay $(-)$ and in the concrete layer (--).

permeability functions (27) and (28) exhibited in Figure 2, are set to

$$
n_{\mathrm{r}}=\left\{\begin{array}{ll}
1.54, & \text { in } \Omega_{\mathrm{cc}}, \\
1.49, & \text { in } \Omega_{\mathrm{ox}},
\end{array} \quad p_{\mathrm{r}}=\left\{\begin{array}{ll}
2 \cdot 10^{6} \mathrm{~Pa}, & \text { in } \Omega_{\mathrm{cc}}, \\
15 \cdot 10^{6} \mathrm{~Pa}, & \text { in } \Omega_{\mathrm{ox}},
\end{array} s_{\mathrm{r}}^{\ell}=\left\{\begin{array}{ll}
0.01, & \text { in } \Omega_{\mathrm{cc}}, \\
0.4, & \text { in } \Omega_{\mathrm{ox}},
\end{array} \quad s_{\mathrm{r}}^{g}=0,\right.\right.\right.
$$

The effective diffusion coefficient $d_{\mathrm{pm}}^{\alpha}$ is computed from the tortuosity model

$$
d_{\mathrm{pm}}^{\ell}=0, \quad d_{\mathrm{pm}}^{g}=\frac{\phi}{\tau^{2}} s^{g} \zeta^{g} d^{g},
$$

with $\tau=2$ and the source term $g_{\mathrm{e}}$ is set to 0 . The liquid pressure, the temperature, the liquid saturation and the water molar fraction in the liquid phase are set, at initial time in the porous-medium domain, to

$$
p^{\ell}=p_{\mathrm{up}}^{\ell}-\zeta^{l} m_{w} g\left(h_{\mathrm{pm}}-z\right), \quad T_{\mathrm{pm}}=T_{\mathrm{pm}}^{0}, \quad s^{\ell}=1, \quad c_{w}^{\ell}=1 \quad \text { at } t=0,
$$

and at the top boundary $\Gamma_{\text {up }}$ of the porous medium, to

$$
p^{\ell}=p_{\mathrm{up}}^{\ell}, \quad T_{\mathrm{pm}}=T_{\mathrm{up}}, \quad s^{\ell}=s_{\mathrm{up}}^{\ell}=1, \quad c_{w}^{\ell}=c_{w, \mathrm{up}}^{\ell}=1, \quad \text { on } \Gamma_{\mathrm{up}},
$$

with

$$
p_{\mathrm{up}}^{\ell}=4 \cdot 10^{6} \mathrm{~Pa}, \quad T_{\mathrm{pm}}^{0}=T_{\mathrm{up}} .
$$

At the output boundary $\Gamma_{\text {out }}$, the gas pressure is set to $p_{\text {out }}=10^{5} \mathrm{~Pa}$ combined with a vanishing molar and temperature diffusion normal flux and with an outflow boundary condition for the RANS flow. The velocity at the input boundary $\Gamma_{\text {in }}$ is defined by the uncoupled turbulent velocity profile $\mathbf{u}^{0}(z)=\left(\begin{array}{c}u^{0}(z) \\ 0\end{array}\right)$ computed from the Prandlt algebraic turbulent model (see [5]) and which is parametrized by the average velocity

$$
u_{\text {in }}=\frac{1}{h_{\mathrm{ff}}} \int_{0}^{h_{\mathrm{ff}}} u^{0}(z) \mathrm{d} z .
$$

The temperature at the input boundary $\Gamma_{\text {in }}$ is fixed to $T_{\text {in }}=303 \mathrm{~K}$, and the input water molar fraction $c_{w, \text { in }}$ corresponds to the relative humidity

$$
H_{\mathrm{r}}=\frac{p_{\text {out }} c_{w, \text { in }}}{p_{\text {sat }}\left(T_{\text {in }}\right)}=0.5
$$

Homogeneous Neumann boundary conditions are used at the remaining boundaries of the domain including a vanishing velocity a the bottom free-flow domain boundary. 
(a) $T_{\mathrm{pm}}^{0}=303 \mathrm{~K}$

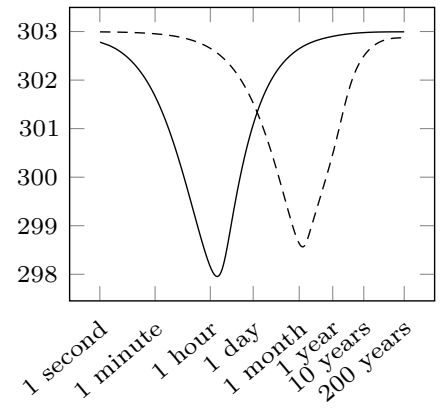

Time (b) $T_{\mathrm{pm}}^{0}=333 \mathrm{~K}$

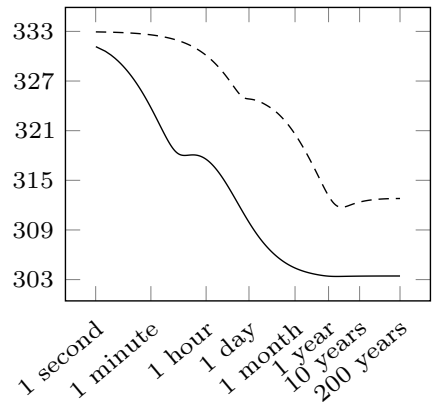

Time

Figure 3 - Average temperature in $\mathrm{K}$ at the interface as a function of time for the input velocities $u_{\text {in }}=5 \mathrm{~m} \cdot \mathrm{s}^{-1}$ in continuous line and $u_{\text {in }}=0.05 \mathrm{~m} \cdot \mathrm{s}^{-1}$ in dashed line and for both initial temperatures in the porous-medium domain.

(a) $T_{\mathrm{pm}}^{0}=303 \mathrm{~K}$

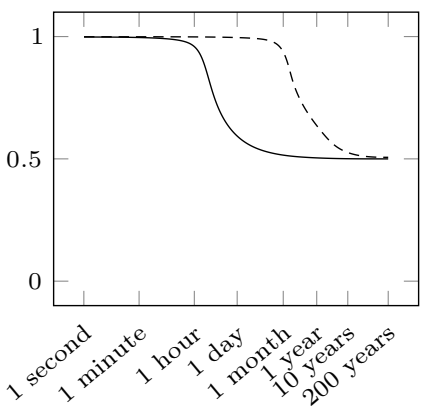

Time (b) $T_{\mathrm{pm}}^{0}=333 \mathrm{~K}$

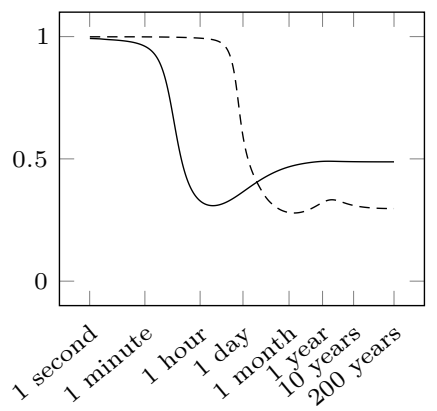

Time

Figure 4 - Average relative humidity at the interface as a function of time for the input velocities $u_{\text {in }}=5 \mathrm{~m} \cdot \mathrm{s}^{-1}$ in continuous line and $u_{\text {in }}=0.05 \mathrm{~m} \cdot \mathrm{s}^{-1}$ in dashed line and for both initial temperatures in the porous-medium domain.

In the following, we consider four test cases defined by two choices of the input gas velocity $u_{\text {in }}=5 \mathrm{~m} \cdot \mathrm{s}^{-1}$ and $u_{\text {in }}=0.05 \mathrm{~m} \cdot \mathrm{s}^{-1}$ and two choices of the initial and top temperature in the porous medium $T_{\mathrm{pm}}^{0}=T_{\mathrm{up}}=303 \mathrm{~K}$ and $T_{\mathrm{pm}}^{0}=T_{\mathrm{up}}=333 \mathrm{~K}$.

The time steps are set by (29) with $\Delta t_{0}=1 \mathrm{~s}, \rho_{\delta t}=1.02$ and $\Delta t_{\max }=1$ year which is reached at the 873th time step at $t=51,7$ year. The time integration reaches the final time $t_{\mathrm{f}}=200$ year after $n_{t}=1022$ time steps. The stopping criteria of the domain decomposition algorithm (15) is set to $\varepsilon=10^{-6}$. For these test cases, no failure of convergence is observed for the nonlinear solvers used for the subproblems, nor for the domain decomposition method.

The Cartesian mesh of the domain is uniform in the $x$ direction with 100 edges. In the $z$ direction, the mesh is refined at both sides of the interface $\Gamma$ with respectively 121 and 162 edges in the porous-medium and free-flow domains including 41 edges in each boundary layer. The mesh step in the $z$ direction varies from $1.27 \mathrm{~mm}$ to $0.11 \mathrm{~m}$ in the porous-medium domain and from $0.057 \mathrm{~mm}$ to $0.05 \mathrm{~m}$ in the free-flow domain, down to the scale of the turbulent boundary layer.

Figures 3, 4 and 5 show respectively the average temperature, the average relative humidity and the average evaporation rate at the interface as a function of time. Solid lines and dashed lines show respectively the results for the input velocity $u_{\text {in }}=5 \mathrm{~m} \cdot \mathrm{s}^{-1}$ and $u_{\text {in }}=0.05 \mathrm{~m} \cdot \mathrm{s}^{-1}$. For both test 
(a) $T_{\mathrm{pm}}^{0}=303 \mathrm{~K}$

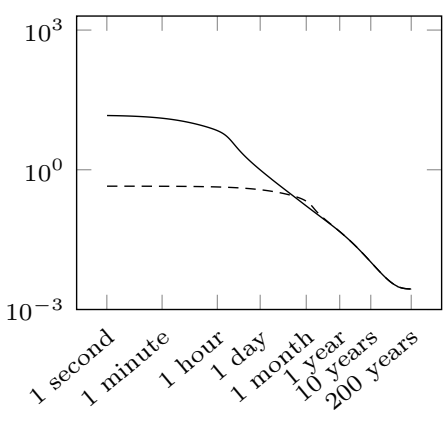

Time (b) $T_{\mathrm{pm}}^{0}=333 \mathrm{~K}$

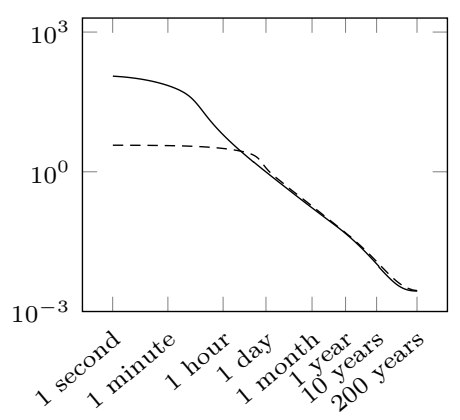

Time

Figure 5 - Average evaporation rate at the interface in $\mathrm{L} \cdot$ day $^{-1} \cdot \mathrm{m}^{-1}$ as a function of time for the input velocities $u_{\text {in }}=5 \mathrm{~m} \cdot \mathrm{s}^{-1}$ in solid line and $u_{\text {in }}=0.05 \mathrm{~m} \cdot \mathrm{s}^{-1}$ in dashed line and for both initial temperatures in the porous-medium domain.

cases with $T_{\mathrm{pm}}^{0}=303 \mathrm{~K}$, the variations of temperature exhibited in Figure 3a at the interface are only due to the vaporization of the liquid phase. During the first stage of the drying process the temperature decreases of a few degrees. The duration of this first stage depends on the input velocity, with roughly 1 hour for the fast input velocity and 1 month for the slow input velocity. This is the time during which the interface is saturated with water vapor on the free-flow side corresponding to a maximum evaporation rate, as shown in Figure 4a and in Figure 5a. The second stage of the drying process is the drop of the evaporation rate, due to the entry of the gas phase in the porous medium, down to a stationary state with a low evaporation rate. Consequently, during that stage, the temperature at the interface warms up almost back to its initial value.

For $T_{\mathrm{pm}}^{0}=333 \mathrm{~K}$, the average temperature, the relative humidity and the evaporation rate are shown in the Figures 3b, 4b and 5b. In this case, due to a higher evaporation rate at high temperature, as exhibited in Figure 5b, the first stage during which the interface is saturated with water is shorter for both velocities, around $5 \mathrm{~min}$ and 9 hour for the fast and the slow input velocity respectively. Then, the dynamic of the coupling is more complex due to the high variation of temperature at the interface induced both by the evaporation of the liquid phase and by the cooling of the interface by the free-flow. Let us remark that in Figure 4b, for the fast input velocity, the interface is dried after 1 hour but still much hotter than the thermal equilibrium state which is reached after 3 month. During that time, the temperature drop goes on, which lowers the vapor pressure and increases the relative humidity at the interface. Note that the stationary solutions obtained at final time for both input velocities differ due to the thermal equilibrium between the porous-medium top boundary at $T_{\text {up }}=333 \mathrm{~K}$ and the free flow depending on the thermal resistance of the free-flow boundary layer.

Figure 6 shows the total gas volume in the porous medium as a function of time. Note that a larger gas volume is reached at the final time of the simulation for the test case with initial temperature $T_{\mathrm{pm}}^{0}=333 \mathrm{~K}$ and input velocity $u_{\mathrm{in}}=0.05 \mathrm{~m} \cdot \mathrm{s}^{-1}$. It has been checked that this is mainly due to the diffusion term in the porous medium.

The average number of domain decomposition iterations per time step is shown in Figure 7 for different mesh sizes $n_{x} \times n_{z}=100 \times 283,50 \times 143,25 \times 73$ and with a total number of 1022 time steps in all cases. Figure 7 exhibits the very good robustness of the algorithm with respect to the mesh size, the input velocity and the porous medium initial temperature. The average number of iterations necessary for the convergence of the domain decomposition algorithm at the stopping criterion $\varepsilon=10^{-6}$ is nearly insensitive to the mesh size and to the initial temperature, and only slightly higher for the low input velocity than for the high one. 
(a) $T_{\mathrm{pm}}^{0}=303 \mathrm{~K}$

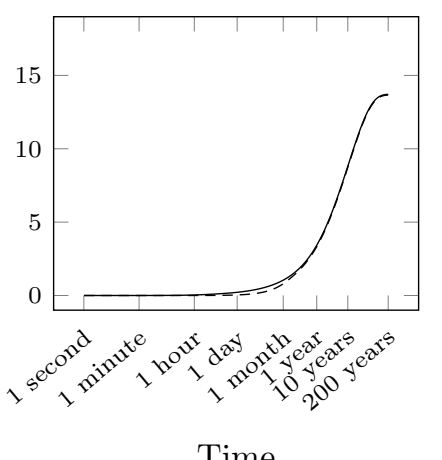

(b) $T_{\mathrm{pm}}^{0}=333 \mathrm{~K}$

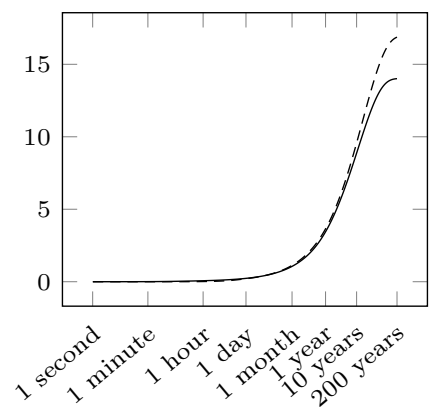

Time

Figure 6 - Average gas volume in the porous medium in $\mathrm{m}^{3}$ as a function of time for the input velocities $u_{\text {in }}=5 \mathrm{~m} \cdot \mathrm{s}^{-1}$ in solid line and $u_{\text {in }}=0.05 \mathrm{~m} \cdot \mathrm{s}^{-1}$ in dashed line and for both initial temperatures in the porous-medium domain.

(a) $T_{\mathrm{pm}}^{0}=303 \mathrm{~K}$

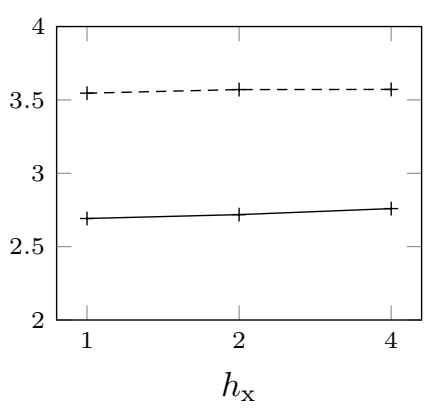

(b) $T_{\mathrm{pm}}^{0}=333 \mathrm{~K}$

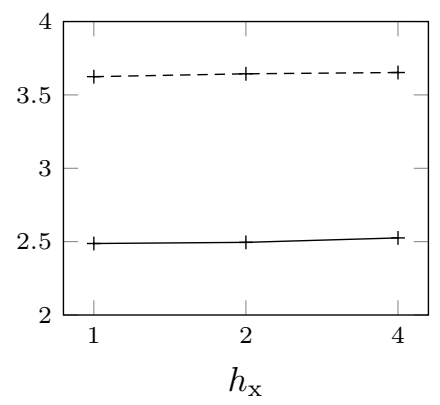

Figure 7 - Average number of domain decomposition iterations per time step as a function of the mesh step in the $x$ direction $h_{\mathrm{x}}(\mathrm{m})$ for the stopping criteria (15) with $\varepsilon=10^{-6}$. 


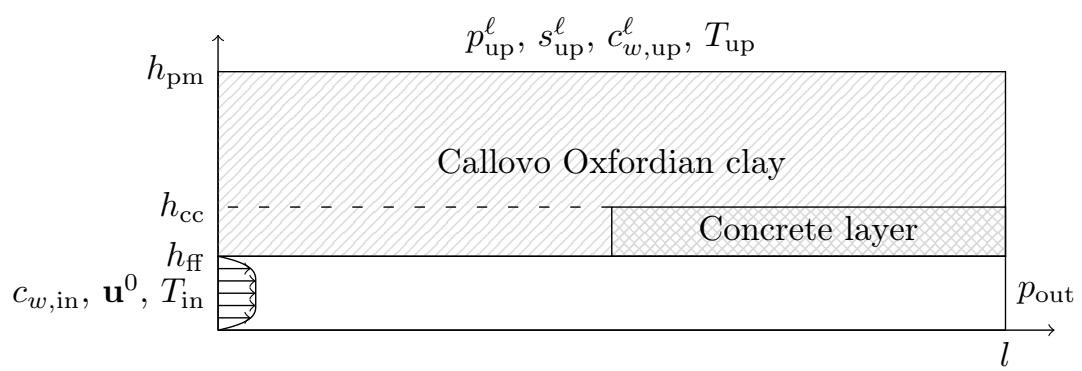

Figure 8 - Setup of the test case for Andra test case 2.

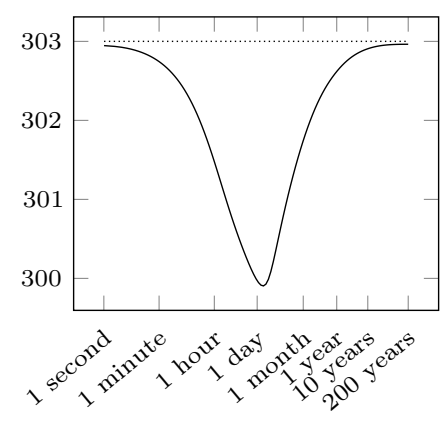

Time

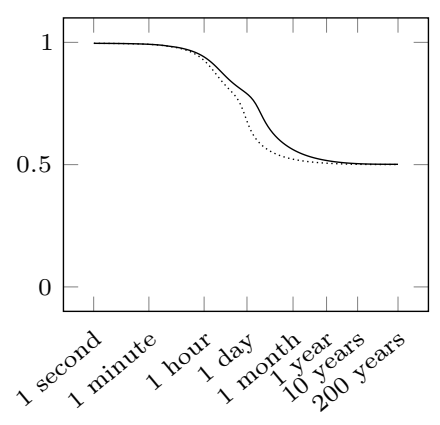

Time

Figure 9 - Average temperature in $\mathrm{K}$ (left) and average relative humidity (right) at the interface as functions of time for the nonisothermal test case $(-)$ and the isothermal test case $(\cdots$.$) .$

\subsection{Andra test case 2}

The objective of this second test case is to test the robustness of the domain decomposition algorithm with respect to the heterogeneities of the porous medium in the direction of the interface $\Gamma$. As exhibited in Figure 8, the concrete layer is now defined by $\Omega_{\mathrm{cc}}=\left(\frac{l}{2}, l\right) \times\left(h_{\mathrm{ff}}, h_{\mathrm{cc}}\right)$. All the physical parameters are the same as in the previous test case except the input velocity set to $u_{\text {in }}=0.5 \mathrm{~m} \cdot \mathrm{s}^{-1}$, and the gas effective diffusion coefficient set to $d_{\mathrm{pm}}^{g}=0$. The initial and top boundary temperatures in the porous medium are set to $T_{\mathrm{pm}}^{0}=T_{\mathrm{up}}=303 \mathrm{~K}$ and the input temperature in the free-flow domain is set to $T_{\text {in }}=303 \mathrm{~K}$.

The Cartesian mesh is uniform in the $x$ direction with 400 edges and refined at both sides of the interface $\Gamma$ with 61 and 82 edges along the $z$ direction respectively in the porous-medium and free-flow domains. In the $z$ direction, 21 edges are used to mesh the boundary layer of each subdomain. The mesh step along the $z$ direction varies from $2.6 \mathrm{~mm}$ to $0.22 \mathrm{~m}$ in the porous-medium domain and from $0.21 \mathrm{~mm}$ to $0.1 \mathrm{~m}$ in the free-flow domain.

The time stepping defined by (29) uses the maximal time step $\Delta t_{\max }=t_{\mathrm{f}}$, the initial time step $\Delta t_{0}=1 \mathrm{~s}$ and the growth rate $\rho_{\delta t}=1.2$. The final time of the simulation is set to $t_{\mathrm{f}}=200$ year and is reached after 115 time steps.

The results are compared to the isothermal counterpart of this test case which has been presented in [6] with no energy conservation and a fixed temperature $T=303 \mathrm{~K}$.

Figures 9 and 10 exhibit for both the isothermal and nonisothermal cases, the average temperature, relative humidity, evaporation rate at the interface and the gas volume in the porous medium as functions of time. The differences between the solutions of both cases are small due to the rather low evaporation rate inducing a low temperature decrease at the interface. It results in a small reduction of the evaporation rate during the first stage of the drying process for the nonisothermal test case compared with the isothermal test case.

The Robin coefficients $L, M_{w}-M_{a}, N$ as functions of $x$ along the interface $\Gamma$ are shown in 


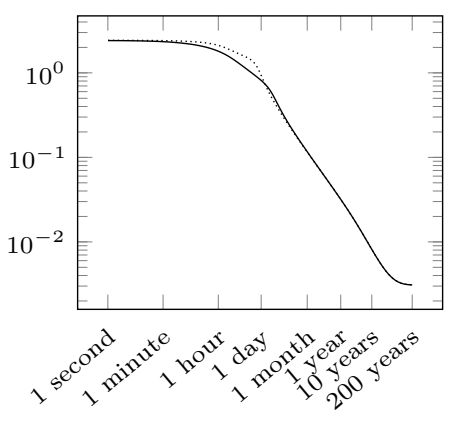

Time

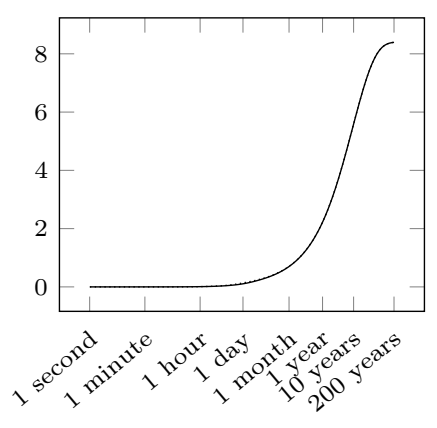

Time

Figure 10 - Average evaporation rate at the interface in $\mathrm{L} \mathrm{day}^{-1} \mathrm{~m}^{-1}$ (left) and average gas volume in the porous medium in $\mathrm{m}^{3}$ (right) as functions of time for the nonisothermal test case (-) and the isothermal test case $(\cdots . \cdots$.

(a) $L, M_{w}-M_{a}, N$

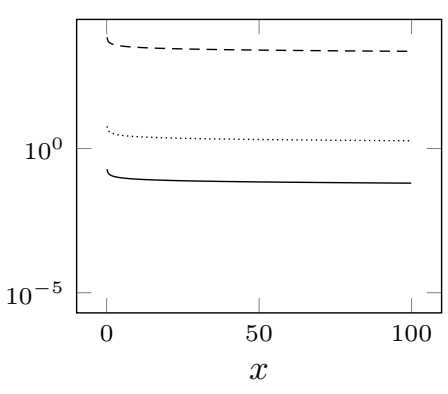

(b) $P, Q$

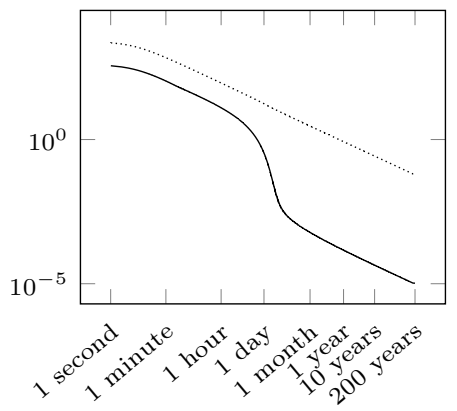

Time

Figure 11 - Value of the Robin coefficients $L(-), M_{w}-M_{a}(--)$ and $N(-)$ along the interface (left) and average value of the Robin coefficients $P(-), Q(\cdots)$ over time (right).

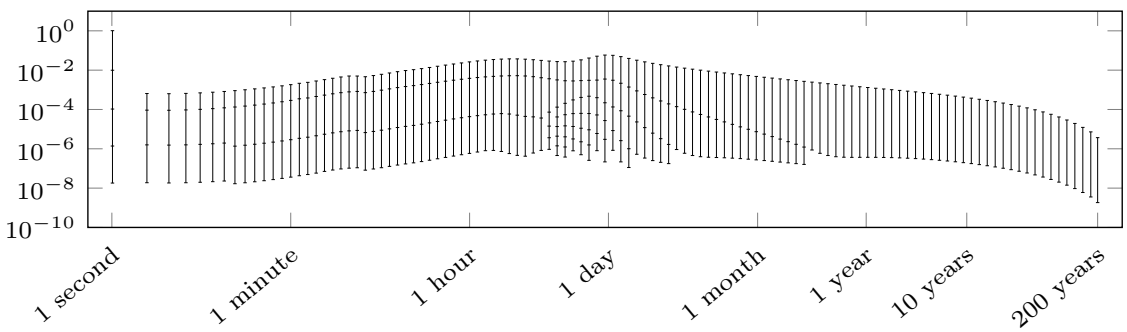

Time

Figure 12 - Convergence of the domain decomposition relative residual (15) at each time step for the isothermal test case. 


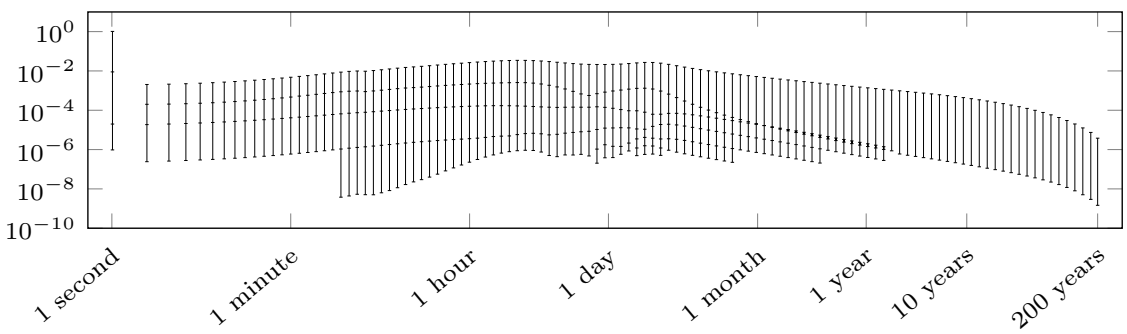

Time

Figure 13 - Convergence of the domain decomposition relative residual (15) at each time step for the nonisothermal test case.

Figure 11. Accordingly with remarks 1 and 2, the coefficients $L$ and $N$ are roughly proportional and the coefficients $L$ and $M_{w}-M_{a}$ are exactly proportional for this data set. Note that, for the Robin coefficients $M_{i}, i \in \mathcal{C}$, only the value of $M_{w}-M_{a}$ matters from the sum to 1 of the gas molar fractions. The average value along the interface $\Gamma$ of the Robin coefficients $P$ and $Q$ are shown as a function of time in Figure 11. Let us stress the dependence of $P$ on the exponentially growing time step and on the relative humidity at the interface. It can be checked that the Robin coefficient $P$ corresponds roughly to a Dirichlet condition at the first stage of the drying process before the drop of the evaporation rate and to a Neumann boundary condition after the drop of the evaporation rate. This adaptation of $P$ to the time step size and to the relative humidity at the interface is crucial to obtain the convergence of the domain decomposition algorithm that could not be obtained with a constant value of $P$. Figures 12 and 13 show the good convergence of the domain decomposition algorithm both for the isothermal and nonisothermal test cases with respectively an average of 3.44 and 4.25 iterations per time step to reach the stopping criteria (15) with $\varepsilon=10^{-6}$.

From remarks 1 and 2, a one parameter family of independent on $x$ Robin coefficients $L, M_{i}$, $i=a, w, N$ can be obtained using $L>0$ as a real parameter and setting

$$
\begin{aligned}
N & =C_{\mathrm{p}, a}^{g} m_{a} L, \\
M_{i} & =h_{i}^{g}\left(T_{\mathrm{in}}\right) L, i=a, w .
\end{aligned}
$$

Figure 14 plots the average number of DDM iterations per time step, as a function of $L$, obtained on a Cartesian mesh of size $25 \times 73$ refined at both sides of the interface. Figure 14 exhibits a plateau of convergence with less than $6 \mathrm{DDM}$ iterations in average in the range $L \in(0.025,0.125)$, then the number of iterations rises very rapidly and the algorithm fails to converge for $L \leq 10^{-5}$ or $L \geq 0.2$. The minimum number of iterations, roughly 4.13, is obtained for $L=0.082$. It is to be compared with the average of 4.22 DDM iterations per time step obtained with the Robin coefficients derived numerically from equations (17), (19), (22). We deduce that independent on $x$ Robin coefficients can be competitive for this test case and also that our computation almost reaches the optimal number of iterations of this one parameter family. A value of $N$ (and hence $L=\frac{N}{C_{\mathrm{P}, a}^{g} m_{a}}$ ) can be obtained using convective heat transfer coefficient correlations which relate the Nusselt number to the Prandtl and Reynolds numbers. Applying the Dittus-Boelter correlation [36] typical for turbulent pipe flow configurations, we obtain $L=0.053$ and an average of 4.7 DDM iterations per time step, which is slightly higher than what is obtained with our methodology.

\subsection{Andra test case 3}

The objective of this third Andra test case is to account for the heat produced by the radioactive waste packages in the disposal. For that purpose, we consider an horizontal 2D cut along the $x$ and $y$ 


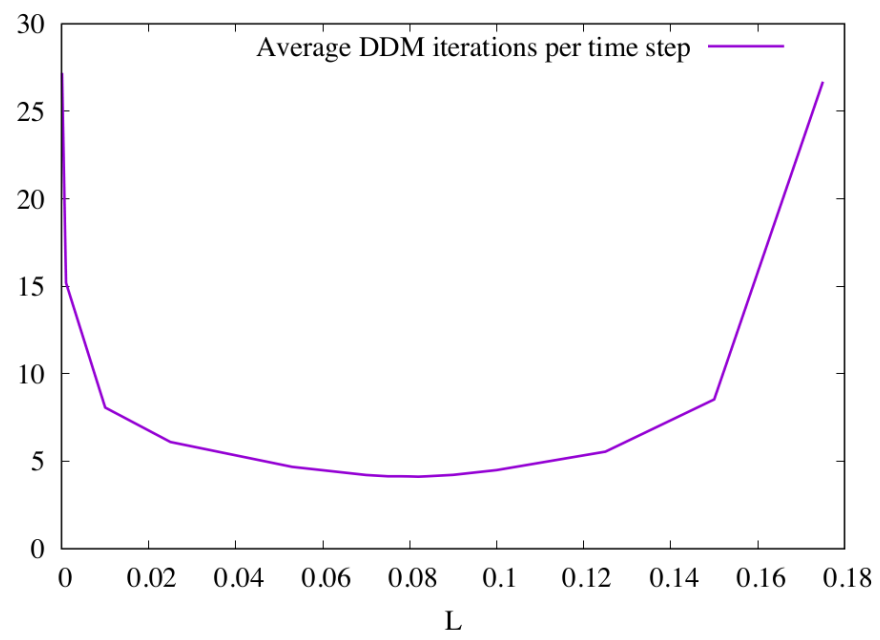

Figure 14 - Average number of DDM iterations per time step as a function of the independent on $x$ Robin coefficient $L$ and using $N=C_{\mathrm{p}, a}^{g} m_{a} L$, and $M_{i}=h_{i}^{g}\left(T_{\mathrm{in}}\right) L, i=a, w$. The mesh is Cartesian of size $25 \times 73$.

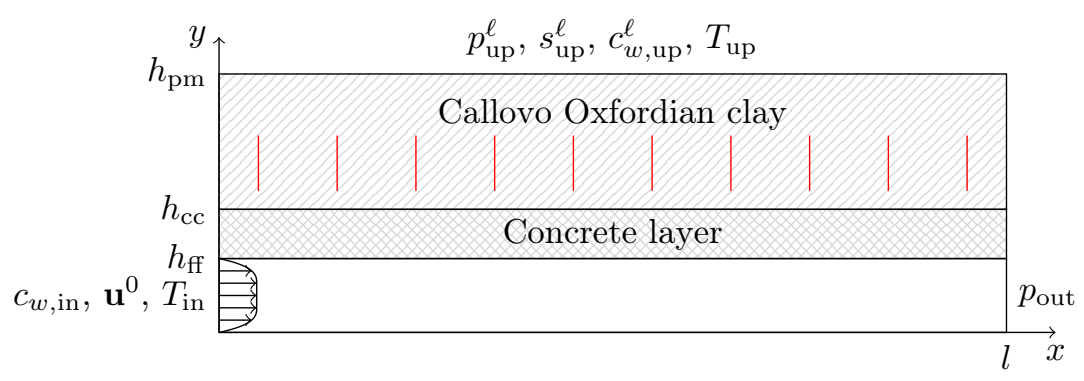

Figure 15 - Setup of the Andra test case 3 with the heat sources in red. 


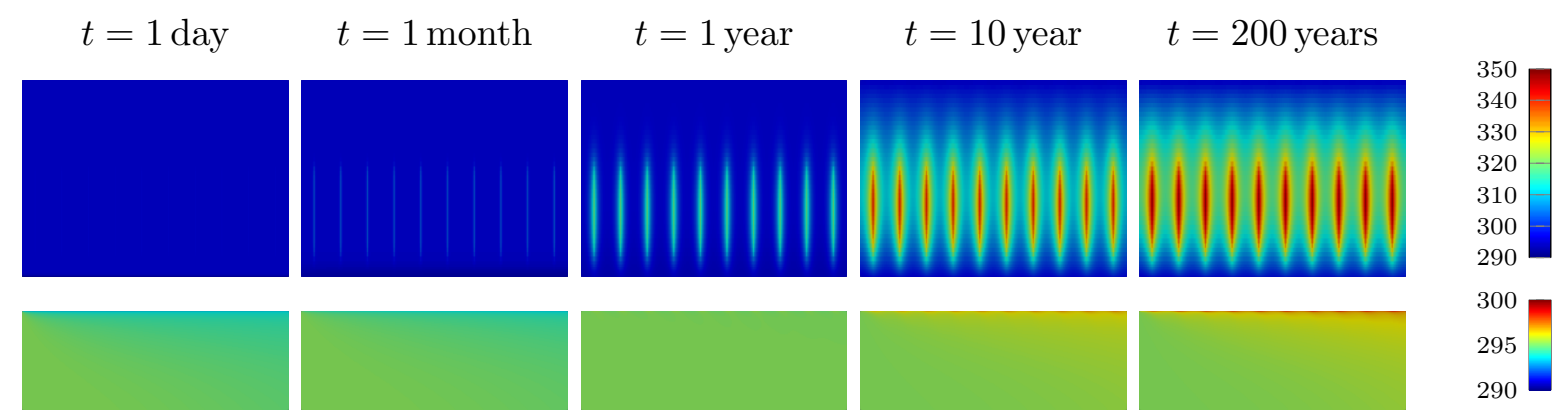

Figure 16 - Temperature in $\mathrm{K}$ in the porous-medium and free-flow domains for the high heat source test case.

directions of the disposal and ventilation gallery. The computational domain is shown in Figure 15 and is similar to the one used for the first test case placed in the horizontal position. The free-flow domain has a length of $l=400 \mathrm{~m}$ and a depth of $h_{\mathrm{ff}}=5.25 \mathrm{~m}$. The depth of the porous-medium domain and of the concrete layer are set to $h_{\mathrm{pm}}=50 \mathrm{~m}$ and $h_{\mathrm{cc}}=6.75 \mathrm{~m}$. Let $\delta l_{\mathrm{s}}=1 \mathrm{~m}$ and $\delta h_{\mathrm{s}}=20 \mathrm{~m}$ be respectively the length and the depth of a heat source. The location of the $n_{\mathrm{s}}=10$ heat sources in the porous-medium domain, as exhibited in Figure 15, is defined by

$$
\Omega_{\mathrm{s}}=\bigcup_{k=1}^{n_{\mathrm{s}}}\left(l_{k}, l_{k}+\delta l_{\mathrm{s}}\right) \times\left(h_{\mathrm{s}}, h_{\mathrm{s}}+\delta h_{\mathrm{s}}\right)
$$

with $l_{k}=40\left(k-\frac{1}{2}\right) \mathrm{m}, k=1, \cdots, 10$, and $h_{\mathrm{s}}=10.25 \mathrm{~m}$. Let us define

$$
\mathrm{g}_{\mathrm{e}}= \begin{cases}\mathrm{g}_{\mathrm{s}} & \text { in } \Omega_{\mathrm{s}}, \\ 0 & \text { in } \Omega_{\mathrm{pm}} \backslash \Omega_{\mathrm{s}}\end{cases}
$$

and consider three different test cases corresponding to either no heat source with $\mathrm{g}_{\mathrm{s}}=0$, or low heat sources with $\mathrm{g}_{\mathrm{s}}=1 \mathrm{~W} \cdot \mathrm{m}^{-2}$, or high heat sources with $\mathrm{g}_{\mathrm{s}}=25 \mathrm{~W} \cdot \mathrm{m}^{-2}$.

The boundary and initial conditions are the same as in the first test case with gravity set to zero, $T_{\mathrm{pm}}^{0}=T_{\mathrm{up}}=296.15 \mathrm{~K}, T_{\mathrm{in}}=295.15 \mathrm{~K}$ and $u_{\mathrm{in}}=0.55 \mathrm{~m} \cdot \mathrm{s}^{-1}$. All the remaining physical parameters are the same as in the first test case.

The time stepping defined by (29) uses the maximal time step $\Delta t_{\max }=1$ year, the initial time step $\Delta t_{0}=1 \mathrm{~s}$ and the growth rate $\rho_{\delta t}=1.2$. The final time of the simulation is set to $t_{\mathrm{f}}=200$ year and is reached after 290 time steps.

The Cartesian mesh has 400 edges along the $x$ direction, and has respectively 61 and 162 edges along the $y$ direction in the porous-medium and in the free-flow domains, including 41 edges for the boundary layer of each domain. The mesh step along the $y$ direction varies from $8.8 \mathrm{~mm}$ to $1 \mathrm{~m}$ in the porous-medium domain and from $0.3 \mathrm{~mm}$ to $0.053 \mathrm{~m}$ in the free-flow domain.

Figure 16 and 17 exhibit respectively, at different times for the high heat source test case, the temperature in the porous-medium and free-flow domains, and the gas saturation in the porous medium and the relative humidity in the free-flow domain. One can observe the effect of the heat source on the desaturation of the porous medium at final time as well as the boundary layers at the interface in the free-flow domain both for the temperature and relative humidity and both at time $t=1$ day and at final time.

Figure 18 exhibits the average temperatures in the porous-medium and the free-flow domains as functions of time. Figures 19 and 20 exhibit the average temperature, relative humidity, evaporation rate at the interface and gas volume in the porous medium as functions of time. It can be checked that the temperature rise in the porous medium occurs during the drop of the evaporation rate. This explains the small differences observed between the different test cases for both the evaporation rate 


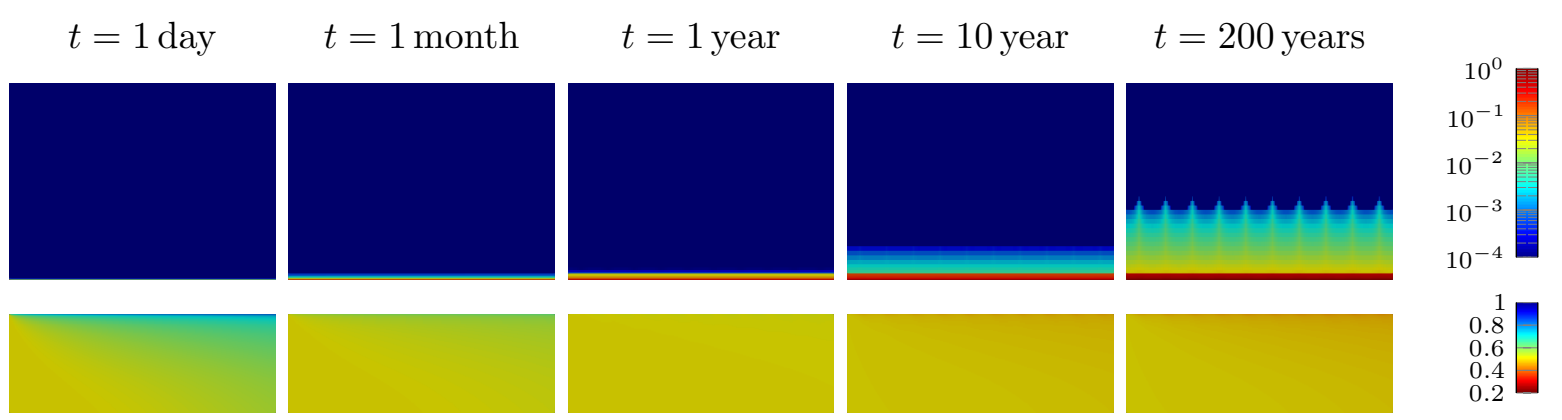

Figure 17 - Gas saturation in the porous-medium domain and relative humidity in the free-flow domain for the high heat source test case.

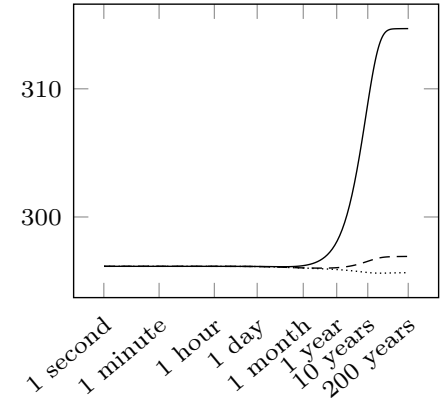

Time

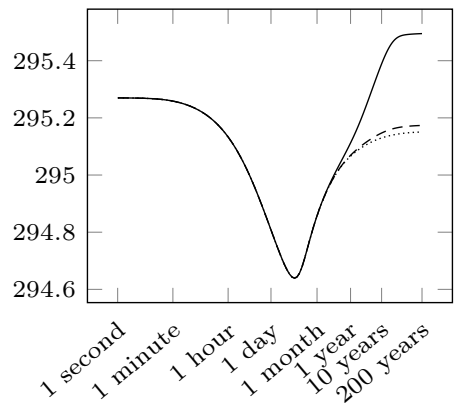

Time

Figure 18 - Average temperature in $\mathrm{K}$ in the porous medium (left) and in the free-flow domain (right) as functions of time and for the test cases: with no heat source $(-)$, with low heat sources $(--)$, with high heat sources $(-)$.

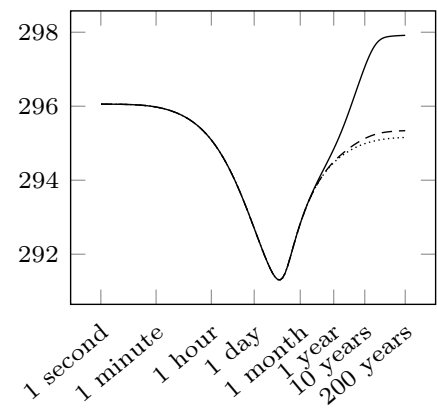

Time

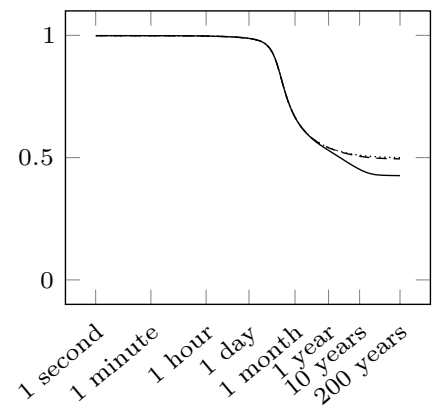

Time

Figure 19 - Average temperature in $\mathrm{K}$ (left) and average relative humidity (right) at the interface as functions of time and for the test cases: with no heat source (...), with low heat sources (--), with high heat sources (-). 


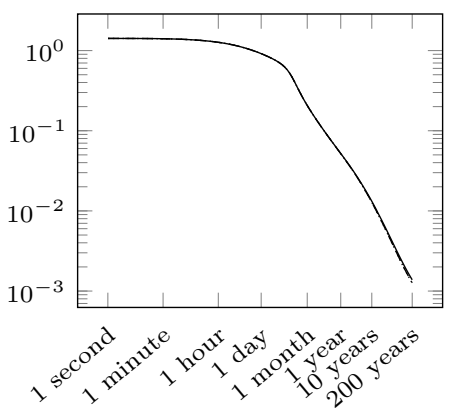

Time

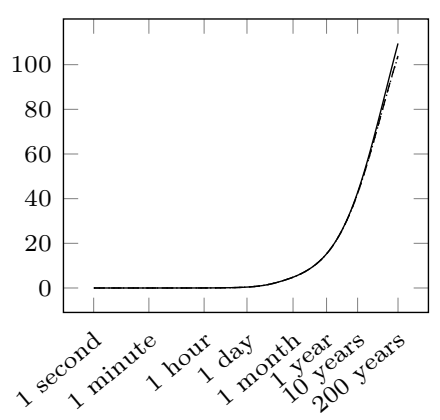

Time

Figure 20 - Average evaporation rate at the interface in $\mathrm{L} \mathrm{day}^{-1} \mathrm{~m}^{-1}$ (left) and average gas volume in the porous medium in $\mathrm{m}^{3}$ (right) as functions of time and for the test cases: with no heat source $(-)$, with low heat sources (--), with high heat sources $(-)$.

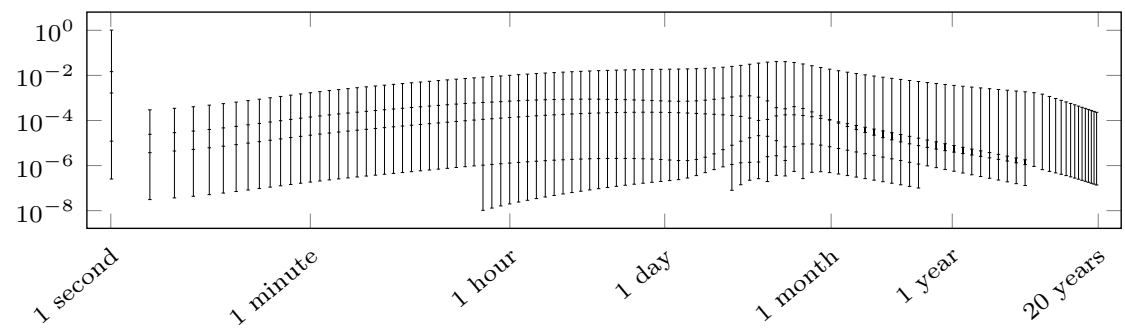

Time

Figure 21 - Convergence of the domain decomposition relative residual (15) at each time step up to $t=20$ years for the no heat source test case.

and the gas volume in Figure 20. These differences appear only at the end of the simulation where the evaporation rate and the gas volume are slightly larger for the high heat source case than for the other cases.

Figures 21 and 22 show the good convergence of the domain decomposition algorithm both with no heat source and with high heat sources. Shortly before 20 years, the time step reaches the maximal time step $\Delta t_{\max }$ and both solutions are close to the stationary solutions. For $t>20$ years, both domain decomposition methods converges in 2 iterations until the final time $t_{\mathrm{f}}=200$ years. Over the overall simulation, the convergence is obtained with respectively an average of 2.84 and 2.94 iterations per time step for the no heat and high heat source test cases. Only one additional iteration for the high heat source test case is needed around $t=1$ year when the heat sources warm up the porous-medium and free-flow domains.

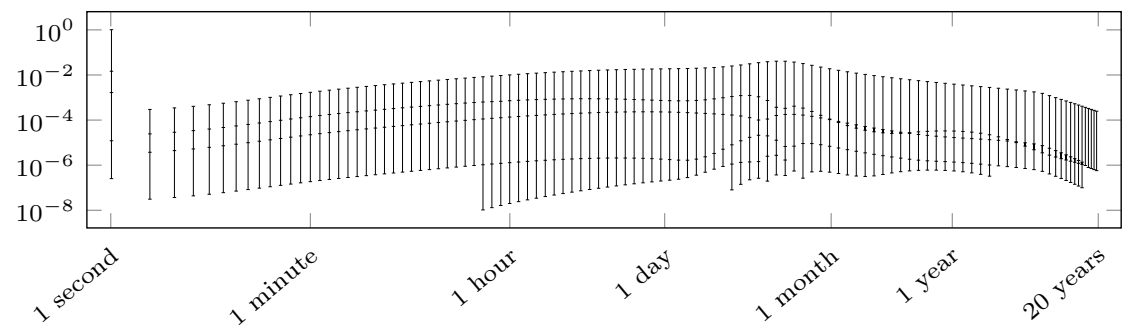

Time

Figure 22 - Convergence of the domain decomposition relative residual (15) at each time step up to $t=20$ years for the high heat source test case. 
(a) Liquid saturation

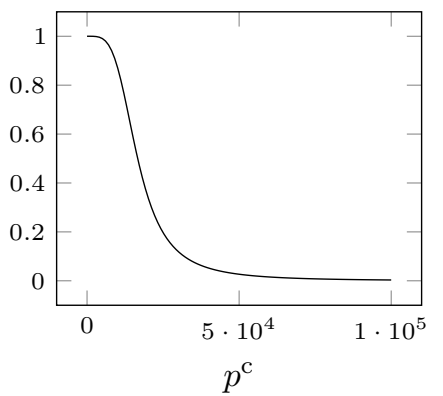

(b) Relative permeability

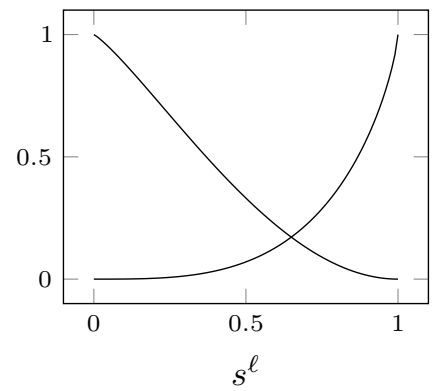

Figure 23 - The liquid saturation as a function of the capillary pressure and the relative permeabilities of both phases as a function of the liquid saturation.

\subsection{Drying test case}

The objective of this last test case is to assess the robustness of the domain decomposition algorithm in the case of a much more permeable porous medium and for a total evaporation of the liquid in the porous medium. For that purpose, we consider the nonisothermal version of the test case introduced in [5, Section 4.4] and compare the solutions and convergence of the domain decomposition method both for the isothermal and nonisothermal test cases.

The setup of the test case is similar to the first test case with dimensions reduced to $l=1 \mathrm{~m}$, $h_{\mathrm{ff}}=0.5 \mathrm{~m}$ and $h_{\mathrm{pm}}=1.5 \mathrm{~m}$. The porous medium contains only one rocktype with Van Genuchten parameters set to

$$
\phi=0.15, \quad \mathbb{K}=10^{-12} \mathrm{~m}^{2},
$$

and

$$
n_{\mathrm{r}}=4, \quad p_{\mathrm{r}}=15 \cdot 10^{3} \mathrm{~Pa}, \quad s_{\mathrm{r}}^{\ell}=0, \quad s_{\mathrm{r}}^{g}=0 .
$$

The liquid saturation and the relative permeability functions are shown in Figure 23. The effective diffusion coefficient is set to $d_{\mathrm{pm}}^{\alpha}=0$ for each phase $\alpha \in \mathcal{P}$ and the source term to $\mathrm{g}_{\mathrm{e}}=0$.

The time stepping defined by (29) uses the maximal time step $\Delta t_{\max }=5$ hour, the initial time step $\Delta t_{0}=10^{-6} \mathrm{~s}$ and the growth rate $\rho_{\delta t}=1.2$. The final time of the simulation is set to $t_{\mathrm{f}}=200$ days and reached in 625 time steps.

In the porous medium, the liquid pressure, the temperature, the liquid saturation and the water molar fraction are given at initial time by (30) with $p_{\mathrm{up}}^{\ell}=10^{5} \mathrm{~Pa}$ and $T_{\mathrm{pm}}^{0}=333 \mathrm{~K}$. As opposed to the previous test cases, an homogeneous Neumann boundary condition is imposed at the top boundary $\Gamma_{\text {up }}$. Together with the vanishing liquid residual saturation, this allows for a total evaporation of the liquid phase from the porous-medium. The input temperature at $\Gamma_{\text {in }}$ is set to $T_{\text {in }}=333 \mathrm{~K}$ and the input velocity to $u_{\text {in }}=1 \mathrm{~m} \cdot \mathrm{s}^{-1}$. The remaining parameters are unchanged compared to the first test case.

The Cartesian mesh is uniform in the $x$ direction with 100 edges and is refined at both sides of the interface $\Gamma$ in the $z$ direction with respectively 121 and 162 edges in the porous-medium and free-flow domains, including 41 edges for the boundary layer of each domain. The mesh step along the $z$ direction varies from $1.3 \mathrm{~mm}$ to $11.2 \mathrm{~mm}$ in the porous-medium domain and from $0.1 \mathrm{~mm}$ to $5 \mathrm{~mm}$ in the free-flow domain.

Figure 24 exhibits the variations of the temperature in the porous medium and in the free-flow domains due to the vaporization of the liquid phase. The gas saturation in the porous medium and the relative humidity in the free-flow domain are shown in Figure 25. During the first 10 days of the simulation, the high vaporization rate lowers the temperature of say $15 \mathrm{~K}$ in the porous medium. At 


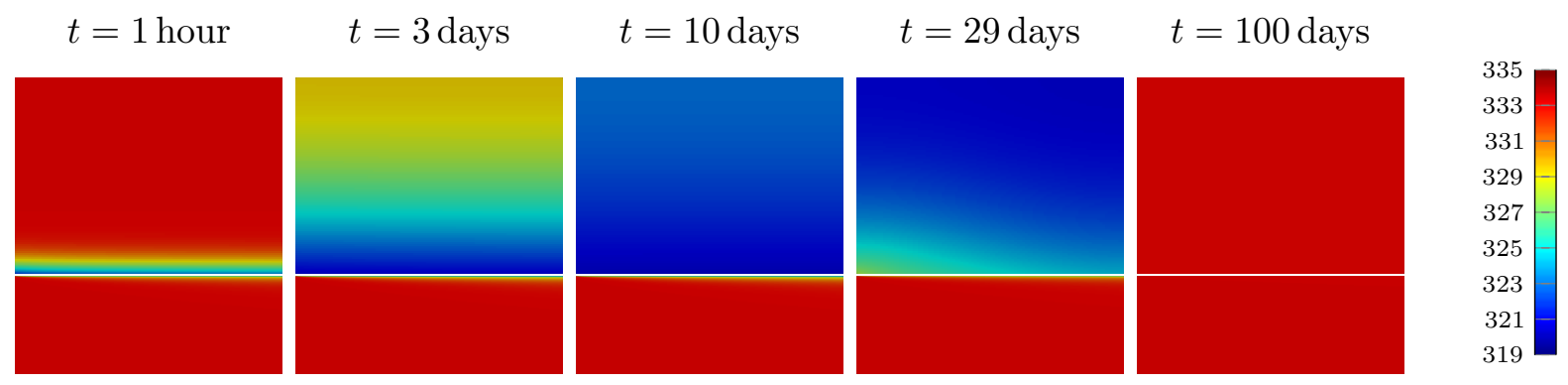

Figure 24 - Temperature in $\mathrm{K}$ in the porous-medium and free-flow domains for the nonisothermal drying test case.

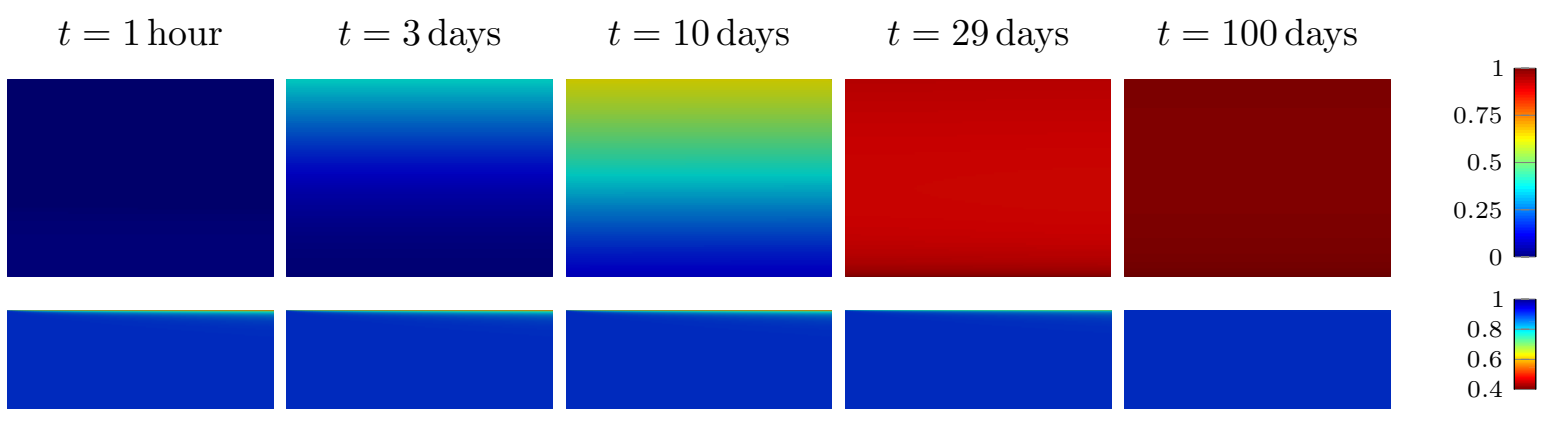

Figure 25 - Gas saturation in the porous-medium domain and relative humidity in the free-flow domain for the nonisothermal drying test case.

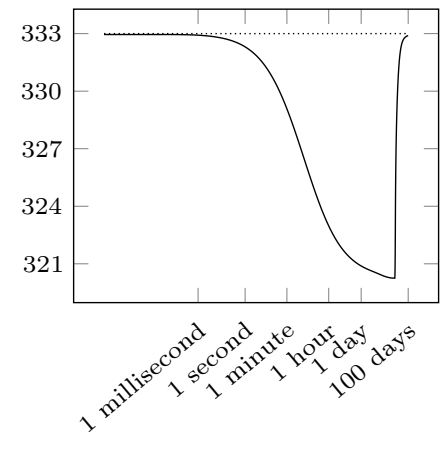

Time

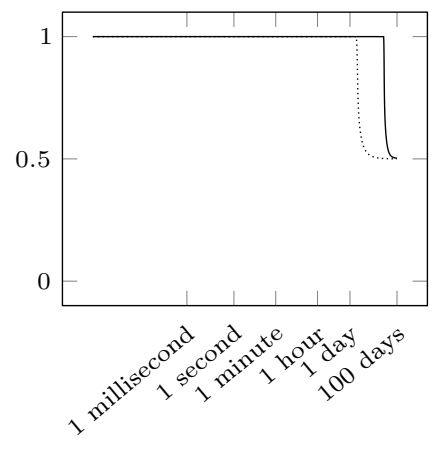

Time

Figure 26 - Average temperature in K (right) and average relative humidity (left) at the interface as functions of time, in dashed line for the isothermal test case and solid line for the nonisothermal test case. 


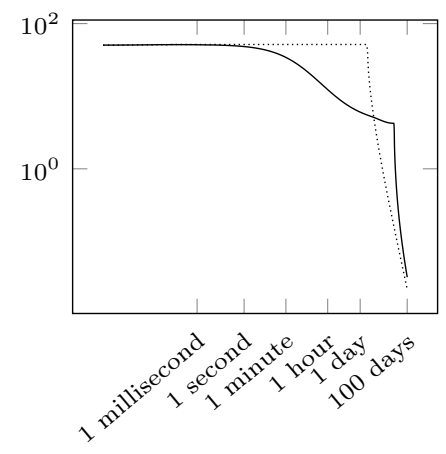

Time

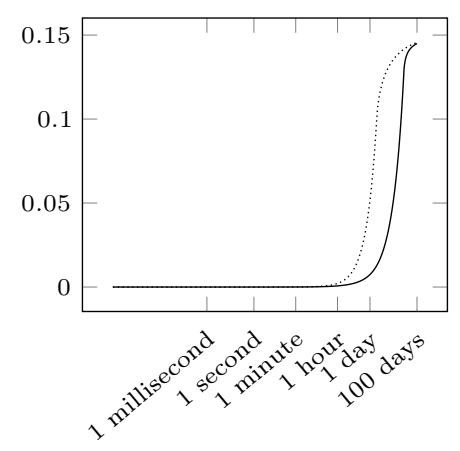

Time

Figure 27 - Average evaporation rate at the interface in $\mathrm{L} \mathrm{day}^{-1} \mathrm{~m}^{-1}$ (left) and average gas volume in the porous medium in $\mathrm{m}^{3}$ (right) as functions of time, in dashed line for the isothermal test case and full line for the nonisothermal test case.

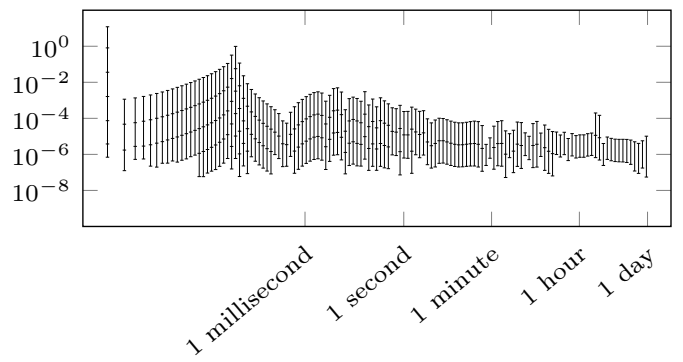

Time

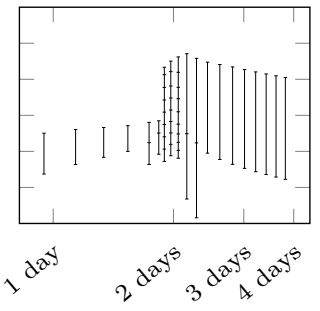

Figure 28 - Convergence of the domain decomposition relative residual (15) at each time step up to $t=4$ days for the isothermal test case.

time $t=3$ days the gas has already started to enter in the porous medium and rises by gravity to the top boundary. At time $t=29$ days, most of the liquid phase is evaporated from the porous medium, the evaporation rate has decreased and the temperature starts to rise in the porous-medium with the ventilation almost back to the input gas temperature at final time $t_{f}=100$ days. The evaporation rate is slow down by the temperature drop in the nonisothermal test case which explains why the drop of the relative humidity and of the evaporation rate appears sooner in the isothermal test case as exhibited in Figures 26 and 27.

Figures 28 and 29 exhibit the convergence of the domain decomposition algorithm showing that it remains efficient both for the isothermal and nonisothermal test cases with respectively an average over the overall simulation of 2.32 and 2.97 iterations per time step to reach the stopping criteria (15)

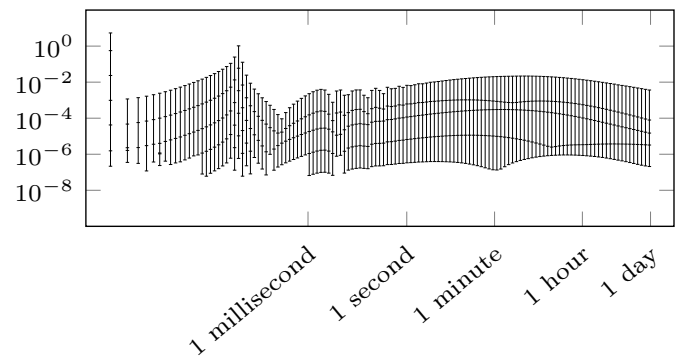

Time

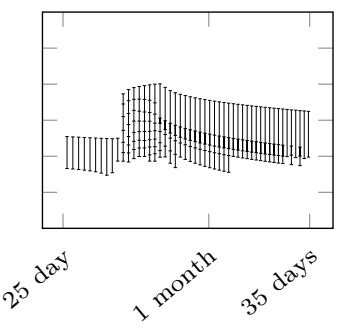

Figure 29 - Convergence of the domain decomposition relative residual (15) at each time step for $t \leq 1$ days and $25 \leq t \leq 35$ days for the nonisothermal test case. 


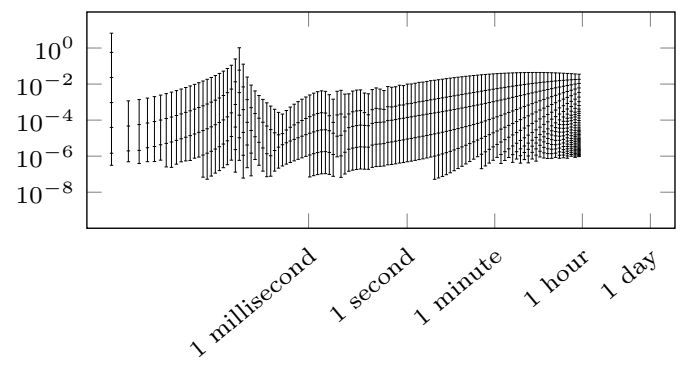

Time

Figure 30 - Convergence of the domain decomposition relative residual (15) at successive time steps for the nonisothermal drying test case with the Robin coefficients $M_{w}$ and $M_{a}$ set to 0 .

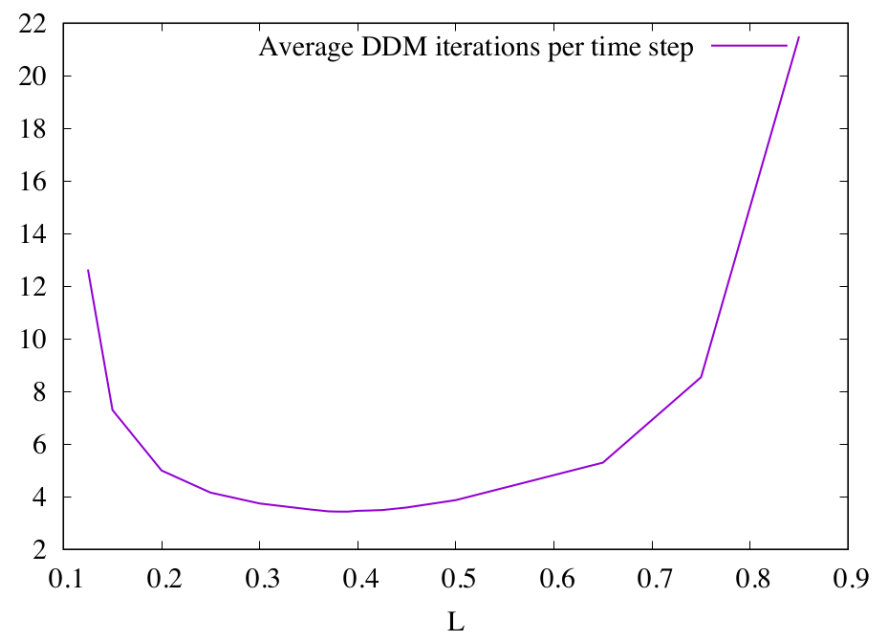

Figure 31 - Average number of DDM iterations per time step as a function of the independent on $x$ Robin coefficient $L$ using $N=C_{\mathrm{p}, a}^{g} m_{a} L$, and $M_{i}=h_{i}^{g}\left(T_{\mathrm{in}}\right) L, i=a, w$. The mesh is Cartesian of size $25 \times 73$.

with $\varepsilon=10^{-6}$. Figure 30 exhibits that the domain decomposition method does not converge if the non diagonal Robin coefficients $M_{i}, i \in \mathcal{C}$ are set to 0 , which exhibits the strong coupling between the transport of energy and of the molar fractions in the boundary layer of the free-flow domain.

As in subsection 5.2, we consider the one parameter family of Robin coefficients defined by the independent on $x$ coefficient $L>0$ and $M_{i}, i=a, w, N$ defined by (31). Figure 31 plots the average number of DDM iterations per time step, as a function of $L$, obtained on a Cartesian mesh of size $25 \times 73$ refined at both sides of the interface. Figure 31 exhibits a plateau of convergence with less than 5 DDM iterations in average in the range $L \in(0.2,0.65)$, then the number of iterations rises very rapidly and the algorithm fails to converge for $L \leq 0.1$ or $L \geq 0.9$. The minimum number of iterations, roughly 3.44, is obtained for $L=0.39$. It is to be compared with the average of 3.0 DDM iterations per time step obtained with the Robin coefficients derived numerically from equations (17), (19), (22). We deduce that, for this test case, our computation is better than the optimal number of DDM iterations of this one parameter family. Applying the Dittus-Boelter correlation for $N$ and (31), we obtain $L=0.136$ for which the algorithm converges in an average of 9.3 iterations which is 3 times higher than what is obtained with our methodology. 


\section{Conclusion and perspectives}

A domain decomposition method to couple nonisothermal compositional gas liquid Darcy and free gas flow and transport have been introduced. Compared with monolithic fully coupled algorithms, it leads to simpler nonlinear and linear systems for which on-the-shelves solvers are available and still provides the fully coupled solution. It also allows to solve the coupled problem using existing codes separately in each subdomain and possibly non-matching meshes at the interface between the porous-medium and free-flow domains.

The efficiency and the robustness of our algorithm with respect to the mesh size, the gas velocity, the porous-medium heterogeneities and the temperature range have been exhibited on 2D test cases with simple geometry. The first three test cases are based on Andra data sets derived from lab experiments and in accordance with the deep disposal center for French radioactive waste project. The fourth test case considers the convective drying of a porous medium with a much larger permeability and the full evaporation of the liquid phase in the porous medium.

The perspectives are to test the domain decomposition algorithm on more complex 3D geometries using a code coupling strategy. The efficiency of the algorithm could also possibly be further improved by designing adaptive stopping criteria for instance based on a posteriori estimates or also by using more advanced techniques to compute the Robin coefficients partially based on the optimization of the convergence rate.

\section{Acknowledgement}

The authors would like to thank Andra, the french national radioactive waste management agency, for supporting this work.

\section{References}

[1] P. Verboven, D. Flick, B. Nicolaï, and G. Alvarez, "Modelling transport phenomena in refrigerated food bulks, packages and stacks: basics and advances," International Journal of Refrigeration, vol. 29 , no. 6, pp. 985--997, 2006.

[2] R. Younsi, D. Kocaefe, S. Poncsak, and Y. Kocaefe, "Computational modelling of heat and mass transfer during the high-temperature heat treatment of wood.," Applied Thermal Engineering, vol. 27, pp. 1424--1431, 2007.

[3] V. Jambhekar, R. Helmig, N. Schroëder, and N. Shokri, "Free-flow-porous-media coupling for evaporation-driven transport and precipitation of salt in soil.," Transport in Porous Media, vol. 110, no. 2, pp. 251--280, 2015.

[4] T. Defraeye, "Advanced computational modelling for drying processes - a review," Applied Energy, vol. 131, pp. 323 -- 344, 2014.

[5] R. Masson, L. Trenty, and Y. Zhang, "Coupling compositional liquid gas Darcy and free gas flows at porous and free-flow domains interface," Journal of Computational Physics, vol. 321, pp. 708 -- 728, 2016.

[6] N. Birgle, R. Masson, and L. Trenty, "A nonlinear domain decomposition method to couple compositional gas liquid darcy and free gas flows," in Proceedings of the Finite Volumes for Complex Applications VIII conference, FVCA 8, Lille, France, June 2017, pp. 387--395, Springer International Publishing, 2017. 
[7] K. Mosthaf, K. Baber, B. Flemisch, R. Helmig, A. Leijnse, I. Rybak, and B. Wohlmuth, "A coupling concept for two-phase compositional porous-medium and single-phase compositional free flow," Water Resources Research, vol. 47, no. 10, 2011.

[8] K. Baber, K. Mosthaf, B. Flemisch, R. Helmig, S. Müthing, and B. Wohlmuth, "Numerical scheme for coupling two-phase compositional porous-media flow and one-phase compositional free flow," IMA Journal of Applied Mathematics, vol. 77, no. 6, p. 887, 2012.

[9] G. S. Beavers and D. D. Joseph, "Boundary conditions at a naturally permeable wall," Journal of Fluid Mechanics, vol. 30, no. 1, p. 197-207, 1967.

[10] T. Defraeye, B. Blocken, and J. Carmeliet, "Analysis of convective heat and mass transfer coefficients for convective drying of a porous flat plate by conjugate modelling," International Journal of Heat and Mass Transfer, vol. 55, no. 1-3, pp. 112 -- 124, 2012.

[11] T. Defraeye, Convective Heat and Mass Transfer at Exterior Building Surfaces. PhD thesis, Catholic University of Leuven, 2011.

[12] I. Rybak, J. Magiera, R. Helmig, and C. Rohde, "Multirate time integration for coupled saturated/unsaturated porous medium and free flow systems," Computational Geosciences, vol. 19, pp. 299--309, 2015.

[13] R. Helmig, B. Flemisch, M. Wolff, A. Ebigbo, and H. Class, "Model coupling for multiphase flow in porous media," Advances in Water Resources, vol. 51, pp. 52 -- 66, 2013. 35th Year Anniversary Issue.

[14] K. Mosthaf, Modeling and Analysis of Coupled Porous-Medium and Free Flow with Application to Evaporation Processes. PhD thesis, University of Stuttgart, 2013.

[15] T. Fetzer, K. Smits, and R. Helmig, "Effect of turbulence and roughness on coupled porous-medium/free-flow exchange processes," Transport in Porous Media, vol. 114, no. 2, pp. 395 -- 424, 2016.

[16] M. Discacciati, A. Quarteroni, and V. Alberto, "Robin-Robin domain decomposition methods for the Stokes-Darcy coupling," SIAM Journal on Numerical Analysis, vol. 45, no. 3, pp. 1246--1268, 2007 .

[17] Y. Cao, M. Gunzburger, X. He, and X. Wang, "Robin-Robin domain decomposition methods for the steady-state Stokes-Darcy system with the Beavers-Joseph interface condition," Numerische Mathematik, vol. 117, pp. 601--629, 2011.

[18] M. Discacciati, E. Miglio, and A. Quarteroni, "Mathematical and numerical models for coupling surface and groundwater flows," Applied Numerical Mathematics, vol. 43, no. 1, pp. 57 -- 74, 2002.

[19] O. Angelini, C. Chavant, E. Chénier, R. Eymard, and S. Granet, "Finite volume approximation of a diffusion-dissolution model and application to nuclear waste storage," Mathematics and Computers in Simulation, vol. 81, no. 10, pp. 2001--2017, 2011.

[20] R. Masson, L. Trenty, and Y. Zhang, "Formulations of two phase liquid gas compositional Darcy flows with phase transitions," International Journal on Finite Volumes, vol. 11, p. 34, 2014. 
[21] A. Lauser, C. Hager, R. Helmig, and B. Wohlmuth, "A new approach for phase transitions in miscible multi-phase flow in porous media," Advances in Water Resources, vol. 34, no. 8, pp. 957 -- 966, 2011.

[22] R. Millington and J. Quirk, "Permeability of porous solids," Nature, vol. 183, pp. 387--388, 1959.

[23] R. Millington, "Gas diffusion in porous media," Science, vol. 130, no. 3367, pp. 100--102, 1959.

[24] M. Gander, "Optimized Schwarz methods," SIAM J. of Numerical Analysis, vol. 44, no. 2, pp. 699--731, 2006.

[25] C. Japhet, F. Nataf, and F. Rogier, "The optimized order 2 method: Application to convection-diffusion problems," Future Generation Computer Systems, vol. 18, no. 1, pp. 17 -30, 2001.

[26] O. Dubois and M. Gander, "Optimized Schwarz methods for a diffusion problem with discontinuous coefficient," Numerical Algorithms, vol. 69, no. 1, pp. 109--144, 2015.

[27] E. Flauraud, I. Faille, N. Willien, F., F., and F. Schneider, "Implicit scheme and domain decomposition method for multiphase flow in porous medium," in Proceedings of the rth European Conference on the Mathematics of Oil Recovery (ECMOR VII), Baveno, Italy, September 5-8 2000, EAGE, 2000.

[28] V. Dolean, P. Jolivet, and F. Nataf, An Introduction to Domain Decomposition Methods: Algorithms, Theory, and Parallel Implementation. SIAM, 2015.

[29] P. Pucci and J. Serin, "The strong maximum principle revisited," Journal of differential equations, vol. 196, no. 1, pp. 1--66, 2004.

[30] J. Vanderborght, T. Fetzer, K. Mosthaf, K. Smits, and R. Helmig, "Heat and water transport in soils and across the soil-atmosphere interface: 1 . theory and different model concepts," Water Resources Research, vol. 53, no. 2, pp. 1057--1079, 2017.

[31] E. Schmidt, "Properties of water and steam in S.I. units," in , Springer-Verlag, 1969.

[32] D. W. Peaceman, Fundamentals of Numerical Reservoir Simulation. Developments in Petroleum Science, Elsevier, 1977.

[33] F. H. Harlow and J. E. Welch, "Numerical calculation of time-dependent viscous incompressible flow of fluid with free surface," Physics of Fluids, vol. 8, no. 12, pp. 2182--2189, 1965.

[34] J. Demmel, S. Eisenstat, J. Gilbert, X. Li, and J. Liu, "A supernodal approach to sparse partial pivoting," SIAM J. Matrix Analysis and Applications, vol. 20, no. 3, pp. 720 -- 755, 1999.

[35] X. Li, J. Demmel, J. Gilbert, L. Grigori, M. Shao, and I. Yamazaki, "Technical report LBNL-44289, Lawrence Berkeley National Laboratory, SuperLU Users' Guide, September," tech. rep., 1999.

[36] F. Dittus and L. Boelter, "Heat transfer in automobile radiator of the tubular type," University of California at Berkley Publ. Eng., vol. 2, pp. 443--461, 1930. 